Universidade de São Paulo

Instituto de Física

\title{
Estudo Analítico e Soluções Exatas da Equação de Spin
}

\author{
Mario Cesar Baldiotti
}

Orientador: Prof. Dr. Dmitri M. Gitman (IF/USP)

\begin{abstract}
Dissertação apresentada ao IFUSP para a obtenção do grau de Doutor em Ciências.
\end{abstract}

\section{Banca Examinadora:}

Prof. Dr. Dmitri M. Gitman (IF/USP)

Prof. Dr. Josif Frenkel (IF/USP)

Prof. Dr. Celso Luiz Lima (IF/USP)

Prof. Dr. Bruto Max Pimentel Escobar (IFT/UNESP)

Prof. Dr. Jéferson de Lima Tomazelli (FGE/UNESP)

São Paulo 


\section{Resumo}

O presente trabalho se destina a um estudo detalhado da chamada equação de spin, a qual pode ser utilizada para descrever o comportamento de sistemas de dois níveis. Para campos externos dados por funções reais, esta equação pode ser identificada com uma redução da equação de Pauli para o caso $0+1$ dimensional. Inicialmente, demonstraremos a relação entre esta equação de spin e várias outras equações relacionadas com diversos

problemas em física. Com estas relações, podemos construir novas soluções da equação de spin a partir do conhecimento de soluções exatas destes outros problemas e, por outro lado, estender a aplicação das soluções obtidas. Em seguida, descrevemos a forma geral da solução desta equação, construímos o operador de evolução e resolvemos o problema inverso, i.e., a determinação do campo externo supondo o conhecimento de uma solução. Finalizando, para o importante caso de campos externos reais, desenvolvemos um método de construção de novas soluções a partir de uma solução previamente conhecida, utilizando a chamada transformação de Darboux. Em particular, demonstramos a existência de operadores de entrelaçamento de Darboux, que não violam a estrutura específica dos sistemas de dois níveis, e permitem construir novos campos externos também dados por funções reais. Como resultado destes desenvolvimentos, apresentamos uma série de novas soluções exatas para a equação de spin. 


\begin{abstract}
The aim of the present work is to study in detail the so called spin equation, which can be used to describe the behavior of two-level systems. We recall that, for real external fields, this equation can be treated as a reduction of the Pauli equation to the $0+1$ dimensional case. Initially, we present the relation between the spin equation and some other equations related to different physical problems. With these relations, we construct new solutions to the spin equation from the knowledge of the exact solutions of these other problems and, on the other hand, extend the applicability of the obtained solutions. After that, we describe the general solution of the spin equation, construct the evolution operator and solve the inverse problem, i.e., the construction of the external field from a given supposed solution. Finally, for the important case of real fields, we develop a method to construct new solutions from a previously known one, by the application of the so called Darboux transformation. In particular, we demonstrate the existence of Darboux intertwining operators which do not violate the specific structure of the two-level systems and allow the construction of external fields which are also given by real functions. As a result of all these developments, we present several new sets of exact solutions for the spin equation.
\end{abstract}


Dedico este trabalho aos meus pais,

José Daniel Baldiotti, Rita de Cássia Baldiotti e, especialmente, ao meu irmão

José Carlos Baldiotti.

O homem sonha monumentos

E só ruínas semeia, Para pousada dos ventos; Como os palácios de areia Dos seus brincos infantis, Mal divisa o que apetece, Que tudo se desvanece...

Paulo Eiró, O Sobrado. 


\section{Agradecimentos}

Ao meu orientador, Prof. Dr. Dmitri M. Gitman; ao Prof. Dr. V.G. Bagrov e a todos os professores do Instituto de Física da USP que contribuíram para a minha formação.

Aos membros da banca.

Ao João Luis Meloni Assirati, cuja contribuição em minha carreira foi nada menos que essencial.

Aos inúmeros amigos que fiz no IFUSP, dentre os quais gostaria de destacar, Rodrigo Fresneda, Carlos Molina Mendes, Thiago dos Santos Pereira, Jose Cleriston Campos de Souza, Fabio Paolini, Ronaldo Carlotto Batista, Vinicius de Souza Fernandes, Marcelo Oliveira da Costa Pires, Milton Alexandre da Silva Junior, Alencar Jose de Faria, Ivan Yasuda e Adriana Ramos de Miranda.

Aos meus amigos Carlos Pedro da Silva, Julio Cesar de Lima e Rogério Morelli.

Ao meu primo Hans Jefferson Radke.

À FAPESP pelo suporte financeiro.

À Beatriz Protti Christino, por existir. 


\section{Índice}

1 Introdução $\quad 1$

1.1 A equação de spin . . . . . . . . . . . . . . . . . . 2

1.2 Campos dependentes do tempo ..................... 4

1.3 Aplicações recentes . . . . . . . . . . . . . . . . . 6

1.3.1 Computadores quânticos .................. 6

1.3.2 Fase geométrica .................... 8

2 Desenvolvimentos formais da equação de spin $\quad 12$

2.1 A equação de spin conjugada . . . . . . . . . . . . . . . 13

2.2 Redução do campo externo . . . . . . . . . . . . . . . . . . 14

2.3 Representação vetorial . . . . . . . . . . . . . . 16

3 Equações relacionadas $\quad 19$

3.0.1 Equação de Schrödinger . . . . . . . . . . . . . . 19

3.0 .2 Equação de Euler . . . . . . . . . . . . . . . 20

4 Sobre as soluções da equação de spin $\quad 23$

4.1 Solução geral da equação de spin . . . . . . . . . . . . . . . . 23

4.2 Soluções estacionárias . . . . . . . . . . . . . . . . . . . 24

4.3 A matriz de transformação . . . . . . . . . . . . . . . 25

4.4 O operador de evolução . . . . . . . . . . . . . . . . 27 
5 O problema inverso para a equação de spin

6 Equação de spin simétrica $\quad 31$

6.1 A solução geral e o problema inverso . . . . . . . . . . . . . . . . 32

6.2 Formas lagrangiana e hamiltoniana da equação de spin simétrica . . . . . . 33

7 Soluções exatas da equação de spin 35

7.1 Lista das soluções exatas . . . . . . . . . . . . . . . . . . 37

8 Transformações de Darboux $\quad 44$

8.1 Construção do operador de entrelaçamento . . . . . . . . . . . . . . . 45

8.2 Transformações de Darboux para a equação de spin . . . . . . . . . . . 47

8.3 Exemplos do método de Darboux . . . . . . . . . . . . . . . . 50

8.3 .1 Primeiro exemplo . . . . . . . . . . . . . . . . 50

8.3 .2 Segundo exemplo . . . . . . . . . . . . . . . . 51

8.3 .3 Terceiro exemplo . . . . . . . . . . . . . . . . 52

9 Conclusão $\quad 55$

$\begin{array}{ll}\text { A O problema de autovalores } & 57\end{array}$

B O problema inverso de autovalores $\quad 59$ 


\section{Capítulo 1}

\section{Introdução}

No tratamento da interação de sistemas quânticos com campos eletromagnéticos intensos, é um fato conhecido [1] que uma descrição semiclássica, na qual o campo é tratado classicamente, fornece resultados equivalentes aos obtidos por uma quantização total do problema, desde que a flutuação no número de fótons possa ser negligenciada. Além disto, sistemas quânticos complexos, com um espectro de energia discreto, freqüentemente encontram-se em situações dinâmicas especiais, nas quais apenas dois estados estacionários possuem participação significativa. Nestes casos, podemos restringir o espaço de Hilbert que descreve tal sistema a um espaço bidimensional. Um ótimo exemplo deste procedimento é o tratamento da polarização da molécula de amônia [2]. Tais sistemas de dois níveis encontram uma vasta gama de aplicações em diversos problemas em física, por exemplo, na teoria semiclássica do laser [3]; na descrição de experimentos de absorção ressonante e indução nuclear [4], ou no comportamento de um feixe de moléculas através de uma cavidade imersa em um campo elétrico ou magnético [5]. Aplicações mais recentes deste sistema serão apresentadas, com um certo nível de detalhamento, no final deste capítulo.

Este trabalho se destina a uma análise detalhada dos sistemas de dois níveis através do estudo da chamada equação de spin, bem como da relação entre esta e outras equações encontradas em diferentes problemas em física. Será também apresentada uma série de novas soluções exatas para esta equação, além de um método que permite a obtenção de 
novas soluções.

\subsection{A equação de spin}

Para o caso mais geral, no qual o sistema está sujeito a interações dependentes do tempo, a descrição do sistema pode ser realizada através de um espinor $V(t)$ com duas componentes dependentes do tempo e a dinâmica será dada pela seguinte equação de Schrödinger em $0+1$ dimensão $(\hbar=c=1)$

$$
\begin{aligned}
& i \frac{d V}{d t}=\left[F_{0} \mathbf{I}+(\tilde{\sigma} \mathbf{F})\right] V, V=\left(\begin{array}{c}
v_{1} \\
v_{2}
\end{array}\right), \\
& (\tilde{\sigma} \mathbf{F})=F_{1} \sigma_{1}+F_{2} \sigma_{2}+F_{3} \sigma_{3},
\end{aligned}
$$

onde I é a matriz identidade $2 \times 2, \tilde{\sigma}=\left(\sigma_{1}, \sigma_{2}, \sigma_{3}\right)$ são as matrizes de Pauli

$$
\sigma_{1}=\left(\begin{array}{ll}
0 & 1 \\
1 & 0
\end{array}\right), \sigma_{2}=\left(\begin{array}{rr}
0 & -i \\
i & 0
\end{array}\right), \sigma_{3}=\left(\begin{array}{rr}
1 & 0 \\
0 & -1
\end{array}\right)
$$

$F_{0}(t)$ e $\mathbf{F}(t)=\left(F_{1}, F_{2}, F_{3}\right)$ quatro funções, em geral complexas, dependentes do tempo. Como veremos, o caso com funções $F_{i}(t)$ complexas descreve um possível amortecimento do sistema.

Se $V(t)$ é uma solução da equação $(1.1) \operatorname{com} F_{0}=0$, uma solução $V^{\prime}(t)$ para $F_{0} \neq 0$ pode ser obtida fazendo

$$
V^{\prime}(t)=\exp \left[-i \int_{0}^{t} F_{0}(\tau) d \tau\right] V(t)
$$

o que permite, sem perda de generalidade, fazer $F_{0}=0$ em (1.1). Com isto, o problema geral assume a forma

$$
i \dot{V}=(\tilde{\sigma} \mathbf{F}) V, V=\left(\begin{array}{c}
v_{1}(t) \\
v_{2}(t)
\end{array}\right), \dot{V}=d V / d t
$$


Neste trabalho, iremos nos referir a (1.3) como a equação de spin e a $\mathbf{F}(t)$ como o campo externo. Em alguns casos, o campo externo será apresentado na forma

$$
\begin{aligned}
& \mathbf{F}=\mathbf{K}+i \mathbf{G}, \mathbf{K}=\operatorname{Re} \mathbf{F}, \mathbf{G}=\operatorname{Im} \mathbf{F}, \\
& \mathbf{K}=\left(K_{k}\right), \mathbf{G}=\left(G_{k}\right), k=1,2,3,
\end{aligned}
$$

onde $\mathbf{K}(t)$ e $\mathbf{G}(t)$ são vetores reais.

Uma realização concreta do problema (1.3) seria uma partícula de momento angular total $J=1 / 2$ inserida em um campo magnético (ou elétrico), dependente do tempo, de intensidade $\mathbf{B}(t)(\mathbf{E}(t))$, neste caso identificamos

$$
\mathbf{F}=-\mu \mathbf{B} \quad(\mathbf{F}=-\mu \mathbf{E})
$$

onde $\mu$ é o momento magnético (elétrico) da partícula. Neste caso, a equação de spin pode ser identificada com a equação de Pauli [6] para uma partícula fixa no espaço. Além disto, como mencionado em [2]; "não importa qual seja o problema de dois níveis original, ele sempre poderá ser interpretado como o problema do elétron".

Mesmo no caso mais simples, quando $\mathbf{F}$ é uma constante, o problema acima possui uma infinidade de aplicações. Por exemplo, freqüentemente, em química, as propriedades óticas provenientes do acoplamento entre dois níveis de uma certa molécula por um campo elétrico estático $E_{0}$ são estudadas fazendo $\mathbf{F}_{o p t}=\left(\mu E_{0}, 0, \Delta_{E}\right)$, onde $\Delta_{E}=\left(E_{0}-E_{1}\right) / 2$ é a diferença de energia entre o estado fundamental e o excitado. Outra aplicação intensamente explorada dos modelos de dois níveis estáticos é o estudo dos efeitos de tunelamento em um duplo poço de potencial [7]; neste caso é conveniente expressarmos o campo como $\mathbf{F}_{\text {tun }}=-1 / 2\left(\Delta_{0}, 0, \varepsilon_{0}\right)$, onde $\varepsilon_{0}$ é uma assimetria na energia do estado fundamental de cada poço e $\Delta_{0}$ a energia de interação entre estes estados. Estes dois problemas estão relacionados pelo operador unitário $R=\exp \left(i \pi \sigma_{2} / 4\right)$, pois fazendo $\varepsilon_{0}=2 \mu E_{0}$ teremos $R\left(\tilde{\sigma} \mathbf{F}_{\text {tun }}\right) R^{-1}=\left(\tilde{\sigma} \mathbf{F}_{\text {opt }}\right)$.

Existem várias equações equivalentes, ou de certa forma relacionadas, com a equação de spin. Por exemplo, a conhecida equação de Euler que surge na teoria do giroscópio ou 
na teoria da precessão de um giromagneto clássico em um campo magnético [5]. A equação de spin com um campo externo onde $F_{i}(t), i=1,2$ são puramente imaginários e $F_{3}$ é constante pode ser identificada como um caso degenerado da equação de Zakarov-Shabat, a qual possui um papel importante na teoria dos sólitons [8].

\subsection{Campos dependentes do tempo}

O estudo de sistemas de dois níveis sujeitos a um campo externo dependente do tempo possui uma vasta e longa história. Dentre os trabalhos pioneiros nesta área, podemos citar Rabi [9], onde se considera um sistema de dois níveis imerso em campo magnético circularmente polarizado. Provavelmente, esta é uma das primeiras soluções exatas apresentadas para o problema dependente do tempo. Resultados mais recentes são apresentados em [10].

Para uma partícula de spin $J=1 / 2$ sujeita a um campo magnético constante $B_{0}$ na direção $z$ e um campo circularmente polarizado de intensidade $B_{1}$ no plano $x, y$ temos

$$
\mathbf{F}=-\mu\left(2 B_{1} \cos (\Omega t),-2 B_{1} \sin (\Omega t), B_{0}\right)
$$

Este problema pode ser drasticamente simplificado pelo uso das chamadas coordenadas girantes (rotating coordenates) [4], situação em que se utiliza um sistema de coordenadas que gira junto com o campo, passando a ser um problema estático, e o resultado é transformado novamente para o sistema fixo do laboratório. Este procedimento se mostrou extremamente útil na solução de vários problemas envolvendo campos dependentes do tempo permitindo, no caso geral, reduzir o campo $\mathbf{F}$ a apenas duas componentes não nulas.

Outro problema de fundamental importância, mas que não pode, em geral, ser tratado exatamente como o anterior, é o caso do campo linearmente polarizado [11]. Como exemplo, considere a interação de uma partícula de spin 1/2 com um campo constante $B_{0}$ na direção $z$ e um campo oscilante $B_{1}$ na direção $x$, 


$$
\mathbf{F}=-\mu\left(2 B_{1} \cos (\Omega t), 0, B_{0}\right)
$$

Modelos com este campo são freqüentemente utilizados para o estudo de problemas envolvendo ressonância elétron-spin, ressonância magnética nuclear e espectroscopia de feixe de átomos. Para o campo F dado acima, encontrar uma solução do problema (1.3) não é uma tarefa simples. Entretanto, para campos com $B_{1}$ não muito intensos, com relação a $B_{0}$, soluções podem ser encontradas para regimes próximos à freqüência de ressonância do sistema, ou seja, quando $\Omega$ é muito próximo a $\mu B_{0}$, utilizando o chamado RWA (rotating-wave approximation) [12]. Considerando o campo linearmente polarizado em (1.6) como uma superposição de dois campos circularmente polarizados, $\cos (\Omega t)=[\exp (-i \Omega t)+\exp (i \Omega t)] / 2$, o primeiro termo $\exp (-i \Omega t)$ recebe o nome de rotating-wave, enquanto o termo $\exp (i \Omega t)$ é chamado de anti-rotating-wave. A aproximação RWA consiste em negligenciar a contribuição do anti-rotating-wave na equação de spin, desprezando termos proporcionais a $\exp \left[i\left(\Omega+\mu B_{0}\right)\right]$.

Devido à importância do problema e à dificuldade na obtenção de soluções exatas, vários métodos de aproximação foram desenvolvidos para encontrar soluções de (1.3). Em [13] é desenvolvida uma série perturbativa convergente para um campo na forma

$$
\mathbf{F}=\left(F_{1}, 0, F_{3}\right), F_{1}=\text { const }
$$

com $F_{3}(t)$ uma função periódica. Para campos $\mathbf{F}(t)$ periódicos ou quasi-periódicos, sistemas de dois níveis foram intensamente estudados por inúmeros autores, utilizando várias aproximações diferentes, e.g., expansões perturbativas [14] e, como descrito acima, o método RWA. Para estes campos periódicos, o formalismo de Floquet [15] fornece um poderoso ferramental para o tratamento do problema, especialmente na aplicação de métodos numéricos. Para uma revisão moderna destes métodos veja [7] e suas referências.

Para sistemas em que $\mathbf{F}$ não é periódico, nem quase-periódico, o problema torna-se bastante complicado. Entretanto, em alguns casos especiais, soluções exatas podem ser encontradas. Em [16], são apresentadas soluções exatas para um campo na forma (1.7) 
para funções $F_{3}(t)$ não periódicas dadas por

$$
F_{3}(t)=\frac{r_{0}}{\cosh \tau}, F_{3}(t)=\frac{r_{0}}{T} \tanh \tau+\frac{r_{1}}{T}, \tau=\frac{t}{T},
$$

onde $r_{0}, r_{1}$ e $T$ são constantes reais. Estas funções vão a zero no infinito, o que as torna convenientes para o tratamento de problemas de espalhamento. Em [17] temos a construção de soluções exatas para três novas funções $F_{3}$, entre as quais a mais simples tem a forma

$$
\begin{aligned}
& F_{3}=c_{0}+\frac{2\left(c_{1}^{2}-c_{0}^{2}\right)}{Q+c_{0}}, Q=\left\{\begin{array}{c}
c_{1} \cosh \varphi, c_{1}^{2}>c_{0}^{2} \\
c_{1} \cos \varphi, c_{1}^{2}<c_{0}^{2}
\end{array},\right. \\
& \varphi=2\left(t \sqrt{\left|c_{1}^{2}-c_{0}^{2}\right|}+c_{2}\right), c_{0,1,2}=\text { const. },
\end{aligned}
$$

as demais expressões envolvem complicadas combinações de funções especiais.

\subsection{Aplicações recentes}

\subsubsection{Computadores quânticos}

Além das várias aplicações citadas acima, atualmente o estudo de sistemas de dois níveis tem atraído uma atenção ainda maior devido a sua relação com o desenvolvimento dos chamados computadores quânticos.

A computação clássica se baseia na manipulação de informações codificadas em um sistema binário, ou seja, toda informação a ser processada é armazenada em uma cadeia de zeros e uns e a computação se dá pela manipulação destas cadeias. Cada dígito destas cadeias recebe o nome de bit. De outra forma, temos inicialmente uma seqüência de $n$ bits que, após ser manipulada, fornece como resultado uma nova seqüência de $m$ bits. Esta manipulação recebe o nome de porta lógica (ou simplesmente porta) de $n$ entradas e $m$ saídas. Além disto, a saída desta porta pode ser introduzida em uma outra porta de $m$ entradas e sofrer uma nova manipulação. A este encadeamento de portas lógicas damos o nome de rede computacional (ou simplesmente rede). Assim, 
a informação mais simples que podemos tratar está armazenada em um único bit e as únicas operações possíveis são a identidade, na qual o bit é mantido inalterado, e a inversão, quando invertemos seu valor original. Esta operação de inversão é chamada porta NOT. Em seguida, podemos imaginar uma porta com dois bits de entrada e um de saída, neste caso existem $4^{2}=16$ operações possíveis. Entretanto, nem todas estas operações são independentes e, na verdade, todas podem ser obtidas com uma rede de três portas fundamentais: a NOT, introduzida anteriormente, a porta $A N D$, cujo resultado é o produto dos bits de entrada e a porta $O R$, cuja saída é zero apenas se as entradas forem ambas zero. É um fato conhecido que qualquer manipulação de $n$-bits pode ser implementada através de uma rede que utilize apenas uma dada combinação destas três portas, ou seja, qualquer porta lógica pode ser descrita como um certo encadeamento das portas NOT, AND e OR ${ }^{1}$. Por conta disso, estas recebem o nome de portas universais.

Na computação quântica, o bit é substituído pelo chamado bit quântico ou qubit, que nada mais é do que o estado de um sistema de dois níveis. Diferentemente do bit clássico, restrito aos valores zero e um, o qubit pode se encontrar na superposição dos níveis fundamentais, i.e., um qubit pode assumir o valor:

$$
|\psi\rangle=\alpha|0\rangle+\beta|1\rangle,|\alpha|^{2}+|\beta|^{2}=1
$$

onde $|0\rangle$ e $|1\rangle$ são os níveis do sistema, por exemplo, os estados do spin de um elétron. Um sistema de $n$-qubits é obtido fazendo

$$
|\psi\rangle=\sum_{x \in\{0,1\}^{n}} \alpha_{x}|x\rangle, \sum_{x \in\{0,1\}^{n}}\left|\alpha_{x}\right|^{2}=1,
$$

onde $|x\rangle$ é o produto tensorial de $n$ estados de sistemas de dois níveis, por exemplo, para um sistema de dois qubits poderíamos ter $|\psi\rangle=1 / \sqrt{2}(|01\rangle+|10\rangle) \operatorname{com}|01\rangle=|0\rangle \otimes|1\rangle$.

A computação quântica é definida como a evolução unitária do estado de entrada, ou seja, as portas lógicas clássicas de $n$ entradas são substituídas por operadores unitários agindo no espaço de Hilbert produto $\mathcal{H}^{\otimes n}$; estes operadores são chamados portas lógicas

\footnotetext{
${ }^{1} \mathrm{Na}$ verdade, apenas duas portas são necessárias: NOT, AND ou NOT, OR.
} 
quânticas. O processo é encerrado com a medição do estado final, operação esta, em geral, não unitária e sujeita à interpretação probabilística da mecânica quântica. Diferente de operações clássicas irreversíveis como as AND e OR, em que a entrada não pode ser sempre reconstruída a partir da saída, a unitariedade dos operadores exige que todo processo computacional quântico seja reversível, de forma que todas as portas quânticas possuem o mesmo número de entradas e saídas. Entretanto, sempre podemos utilizar alguns dos qubits de saída para simular qualquer porta clássica [18].

Como na computação clássica, a computação quântica pode ser realizada com um número reduzido de portas universais. Como exemplo, é possível mostrar [18] que qualquer operação unitária agindo em $n$ qubits pode ser implementada através das chamadas porta de Hadamard $(H)$ e da porta de mudança de fase controlada $(B)$, de um e dois qubits, respectivamente, e representadas na base computacional $\{|00\rangle,|01\rangle,|10\rangle,|11\rangle\}$ pelos operadores unitários

$$
H=\frac{1}{\sqrt{2}}\left(\begin{array}{cc}
1 & 1 \\
1 & -1
\end{array}\right), B(\phi)=\left(\begin{array}{cccc}
1 & 0 & 0 & 0 \\
0 & 1 & 0 & 0 \\
0 & 0 & 1 & 0 \\
0 & 0 & 0 & e^{i \phi}
\end{array}\right)
$$

A implementação experimental destas portas lógicas quânticas exige a manipulação destes sistemas de dois níveis pela ação de campos externos. Como exemplo, em [19] é analisada a realização de uma porta quântica de um qubit através da aplicação de um campo na forma (1.5). De modo geral, a evolução destes sistemas de dois níveis pela aplicação destes campos é descrita pela equação de spin. Assim, a análise desta equação é um problema de fundamental importância para a computação quântica.

\subsubsection{Fase geométrica}

Dado um espaço de Hilbert $\mathcal{H}$ de estados quânticos, o espaço de Hilbert projetivo associado $\mathcal{P}=\mathcal{H} / U(1)$, ou espaço dos raios, é o conjunto das classes de vetores múltiplos (por um 
número complexo qualquer) entre si. Assim, a qualquer estado $V \in \mathcal{H}$ podemos associar a classe $\Pi(V)$ de todos os estados $V^{\prime}$ múltiplos de $V$,

$$
\Pi(V)=\left\{V^{\prime}: V^{\prime}=c V, \text { com } c \text { um número complexo }\right\}
$$

Uma classe $\Pi(V)$ é um elemento de $\mathcal{P}$ e o conjunto de todas as classes $\Pi(V)$, quando $V$ percorre todo o $\mathcal{H}$, constitui o espaço de Hilbert projetivo $\mathcal{P}$. Uma trajetória $V(t)$ em $\mathcal{H}$ tem, assim, uma imagem $\Pi(V(t))$ no espaço projetivo $\mathcal{P}$. Uma característica notável de sistemas quânticos é a capacidade do estado $V$ de manter uma memória da trajetória $\Pi(V(t))$ no espaço projetivo $\mathcal{P}$ quando $\Pi(V(t))$ é periódica. Dada uma evolução temporal

$$
i \dot{V}=H V
$$

com um operador hermitiano $H(t)$, a norma de $V$ se conserva e, portanto, periodicidade em $\mathcal{P}$ significa periodicidade a menos de uma fase em $\mathcal{H}$, ou seja, se o período é $\tau$,

$$
V(\tau)=\exp (i \Gamma) V(0)
$$

com uma fase $\Gamma$ real. Definindo $\tilde{V}(t)=\exp [-i f(t)] V, \operatorname{com} f(\tau)-f(0)=\Gamma$, obtemos $\tilde{V}(0)=\tilde{V}(\tau)$ e, substituindo em $(1.10)$,

$$
\frac{d f}{d t}=i\left(\tilde{V}, \frac{d}{d t} \tilde{V}\right)-(V, H V)
$$

Integrando a equação anterior obtemos

$$
\Gamma=i \int_{0}^{\tau}\left(\tilde{V}, \frac{d}{d t} \tilde{V}\right) d t-\int_{0}^{\tau}(V, H V) d t
$$

Nesta expressão, identificamos a fase dinâmica $\gamma_{D}$ e a fase geométrica [20] $\gamma$,

$$
\gamma_{D}=-\int_{0}^{\tau}(V, H V) d t, \gamma=i \int_{0}^{\tau}\left(\tilde{V}, \frac{d}{d t} \tilde{V}\right) d t
$$

Diferente da fase dinâmica, a fase geométrica é invariante por uma reparametrização do tempo $t^{\prime}=f(t)$, o que mostra que esta fase não depende da velocidade com que diferentes partes da trajetória, tanto em $\mathcal{H}$ quanto em $\mathcal{P}$, são percorridas. Esta característica vem 
do fato de que todos os estados obtidos por esta reparametrização representam trajetórias em $\mathcal{H}$ que diferem apenas por uma fase, enquanto a fase geométrica se relaciona apenas com a área limitada pela curva fechada $\Pi(V(t)), t \in[0, \tau]$, no espaço projetivo $\mathcal{P}$.

Para o caso de sistemas de dois níveis, os vetores de estado no espaço projetivo $\mathcal{P}$ podem ser associados com pontos em uma superfície esférica $S^{2}$, chamada esfera de Bloch. Isto pode ser concretizado, escrevendo o estado do sistema como (veja equação (2.32)):

$$
V=\left(\begin{array}{c}
\exp \left[-\frac{i}{2} \varphi(t)\right] \cos \theta(t) / 2 \\
\exp \left[\frac{i}{2} \varphi(t)\right] \sin \theta(t) / 2
\end{array}\right),
$$

e identificando este estado como o ponto com coordenada polar $\theta$ e azimutal $\varphi$ na esfera de Bloch. Para o caso de uma partícula com spin 1/2, podemos visualizar estes pontos como a direção do spin. Da mesma forma, as trajetórias do sistema podem ser traçadas diretamente na superfície desta esfera. Para este sistema de dois níveis, é fácil mostrar que a fase geométrica $\gamma$ pode ser escrita como a seguinte integral em $S^{2}$

$$
\gamma=-\frac{1}{2} \Delta \Omega, \quad \Delta \Omega=\int_{S} \mathbf{n} \cdot d \mathbf{s} .
$$

onde $S$ é a superfície em $S^{2}$ limitada pela trajetória fechada e (veja equação $(2.29)$ )

$$
\mathbf{n}=(V, \tilde{\sigma} V)=(\sin \theta \cos \varphi, \sin \theta \sin \varphi, \cos \theta)
$$

Na expressão (1.15) $\Delta \Omega$ é o ângulo sólido encerrado pela trajetória em $S^{2}$. Obviamente, estas expressões só podem ser obtidas graças ao caráter bidimensional do sistema. Isto mostra porque os sistemas de dois níveis são objetos interessantes para os estudos e aplicações da fase geométrica. Além disto, existe também uma forte relação entre a fase geométrica para sistemas de dois níveis e a computação quântica. Uma área denominada computação quântica geométrica [21] propõe a utilização desta fase para a implementação de uma porta de mudança de fase controlada e, como vimos, a combinação desta porta com a porta de Hadamard fornece um conjunto de portas quânticas universais.

O cálculo da fase geométrica depende da determinação de soluções periódicas da equação de spin. Neste respeito, os resultados a serem desenvolvidos no capítulo 5 sobre "O 
Problema inverso da Equação de Spin" serão de grande valia. Por exemplo, podemos exigir que nosso espinor tenha a forma

$$
V=\exp [i \alpha]\left(\begin{array}{c}
\exp [-i \beta] \cos \varphi \\
\exp [i \beta] \sin \varphi
\end{array}\right),(V, V)=\text { const. }
$$

$\operatorname{com} \alpha, \beta, \varphi$ funções reais do tempo. Este espinor será solução da equação de spin para o campo externo

$$
\begin{aligned}
& F_{1}=-\dot{\varphi} \sin 2 \beta-\dot{\alpha} \cos 2 \beta \sin 2 \varphi, \\
& F_{2}=\dot{\varphi} \cos 2 \beta-\dot{\alpha} \sin 2 \beta \sin 2 \varphi, \\
& F_{3}=\dot{\beta}-\dot{\alpha} \cos 2 \varphi .
\end{aligned}
$$

Assim, podemos garantir a periodicidade das soluções, a menos de uma fase, exigindo a periodicidade das funções $\beta(t)$ e $\varphi(t)$ e calcular a fase geométrica destas soluções utilizando a expressão (1.15). Isto fornece um método simples para determinar a fase geométrica de soluções para vários campos externos diferentes. Este controle sobre a forma das soluções pode também ser amplamente explorado na construção de portas lógicas quânticas. 


\section{Capítulo 2}

\section{Desenvolvimentos formais da equação de spin}

Este capítulo tem por objetivo a introdução de certas notações e o estabelecimento de algumas relações a serem empregadas no decorrer deste trabalho. Primeiramente, mostraremos como é possível escrever um espinor genérico a partir de uma solução particular da equação de spin. Este resultado será utilizado quando tratarmos da solução geral desta equação na seção 4.1 .

Em seguida, apresentamos uma transformação inversível com a qual podemos reduzir o número de componentes do campo externo, além de estabelecer uma equivalência entre soluções da equação de spin para diferentes tipos de campo, a partir de certos casos particulares desta transformação.

No final deste capítulo, apresentamos uma representação vetorial associada a um par de espinores, bem como uma série de relações associadas a este vetor. Várias destas relações representam apenas manipulações algébricas, mas sua ampla utilização neste trabalho justifica sua explicitação. 


\subsection{A equação de spin conjugada}

A equação conjugada obtida a partir da equação de spin (1.3) tem a forma

$$
i \dot{V}^{+}=-V^{+}\left(\tilde{\sigma} \mathbf{F}^{*}\right), V^{+}=\left(\begin{array}{ll}
v_{1}^{*}(t) & v_{2}^{*}(t)
\end{array}\right) .
$$

O produto interno entre dois espinores $U$ e $V$ é definido como:

$$
(U, V)=U^{+} V=\left(u_{1}^{*} v_{1}+u_{2}^{*} v_{2}\right) .
$$

Explicitando-se cada uma de suas componentes, a equação de spin e sua conjugada fornecem o seguinte conjunto de equações acopladas

$$
\begin{aligned}
& i \dot{v}_{1}=F_{3} v_{1}+\left(F_{1}-i F_{2}\right) v_{2}, i \dot{v}_{2}=-F_{3} v_{2}+\left(F_{1}+i F_{2}\right) v_{1}, \\
& i \dot{v}_{1}^{*}=-F_{3}^{*} v_{1}^{*}-\left(F_{1}^{*}+i F_{2}^{*}\right) v_{2}^{*}, i \dot{v}_{2}^{*}=F_{3}^{*} v_{2}^{*}-\left(F_{1}^{*}-i F_{2}^{*}\right) v_{1}^{*} .
\end{aligned}
$$

Introduzindo o espinor anticonjugado $\bar{V}$

$$
\bar{V}=-i \sigma_{2} V^{*}=\left(\begin{array}{c}
-v_{2}^{*} \\
v_{1}^{*}
\end{array}\right),
$$

podemos escrever o sistema (2.4) como uma equação de spin (1.3) com um campo externo complexo conjugado $F^{*}$,

$$
i \dot{\bar{V}}=\left(\tilde{\sigma} \mathbf{F}^{*}\right) \bar{V} .
$$

As seguintes relações são válidas para todo espinor $V$ e seu anticonjugado $\bar{V}(2.5)$ :

$$
\overline{(\bar{V})}=-V,(\bar{V}, \bar{V})=(V, V),(\bar{V}, V)=(V, \bar{V})=0 .
$$

Uma vez que $V \neq 0$ é ortogonal a $\bar{V}$, estes vetores são linearmente independentes. Portanto, qualquer espinor $U$ pode ser representado como

$$
U=(V, V)^{-1}[(V, U) V+(\bar{V}, U) \bar{V}] .
$$

Na verdade, isto implica na relação de completeza

$$
V V^{+}+\bar{V} \bar{V}^{+}=(V, V) I,
$$


onde

$$
V U^{+}=\left(\begin{array}{cc}
v_{1} u_{1}^{*} & v_{1} u_{2}^{*} \\
v_{2} u_{1}^{*} & v_{2} u_{2}^{*}
\end{array}\right), \operatorname{det} V U^{+}=0
$$

\subsection{Redução do campo externo}

Dada uma equação de spin com um campo externo F, em geral com três componentes não nulas, é sempre possível encontrar uma transformação inversível $\hat{T}(t)$ que permite

eliminar uma das componentes de $\mathbf{F}$, gerando, assim, uma equação de spin equivalente com um certo campo externo reduzido $\mathbf{F}^{\prime}$ de apenas duas componentes.

Vamos introduzir a transformação

$$
V^{\prime}=\hat{T} V, \hat{T}(t)=\exp [i \alpha(t)(\tilde{\sigma} \mathbf{l})]
$$

onde $V$ é uma solução da equação de spin (1.3) com o campo externo $\mathbf{F}$, 1 um vetor complexo arbitrário e $\alpha(t)$ uma função complexa arbitrária.

Se $\mathbf{l}^{2} \neq 0$ podemos, sem perda de generalidade, fazer $\mathbf{l}^{2}=1$. Com isto, a matriz $(2.11)$ torna-se

$$
\hat{T}=\cos \alpha+i(\tilde{\sigma} \mathbf{l}) \sin \alpha
$$

Por substituição direta, verifica-se que o espinor $V^{\prime}$ é também uma solução da equação de spin com o campo externo reduzido $\mathbf{F}^{\prime}$,

$$
\mathbf{F}^{\prime}=[\mathbf{F}-\mathbf{l}(\mathbf{F l})] \cos 2 \alpha+[\mathbf{F} \times \mathbf{l}] \sin 2 \alpha+\mathbf{l}(\mathbf{F l}-\dot{\alpha})
$$

Sendo a matriz $\hat{T}$ inversível, as equações de spin com o campo externo $\mathbf{F}$ e com o campo externo $\mathbf{F}^{\prime}$ (2.12) são equivalentes. Podemos agora escolher uma função $\alpha(t)$ que respeite a relação

$$
\dot{\alpha}=\mathbf{F l} \Longrightarrow \mathbf{F}^{\prime} \mathbf{l}=0
$$

Com isto, a projeção de $\mathbf{F}^{\prime}$ na direção de $\mathbf{l}$ se anula e o campo reduzido $\mathbf{F}^{\prime}$ passa a ter apenas duas componentes pertencentes ao plano ortogonal a $\mathbf{l}$. 
Para o vetor $\mathbf{l}$ com $\mathbf{l}^{2}=0$, temos

$$
\hat{T}=1+i \alpha(\tilde{\sigma} \mathbf{l}),
$$

neste caso,

$$
\mathbf{F}^{\prime}=\mathbf{F}+2 \alpha[\mathbf{F} \times \mathbf{l}]+\mathbf{l}\left[2 \alpha^{2}(\mathbf{F l})-\dot{\alpha}\right] .
$$

Para uma escolha apropriada do vetor complexo l, pode-se sempre eliminar uma das duas componentes $\mathbf{K}=\operatorname{Re} \mathbf{F}^{\prime}$ ou $\mathbf{G}=\operatorname{Im} \mathbf{F}^{\prime}$. Entretanto, neste caso, não podemos imaginar $\mathbf{F}^{\prime}$ como um vetor em um plano fixo, diferente do caso com 1 real.

Vamos escolher $\mathbf{l}$ como o vetor unitário na direção $z, \mathbf{l}=(0,0,1)$, e $\alpha$ sendo uma solução da equação

$$
\dot{\alpha}=F_{3} .
$$

Então, o campo externo reduzido $\mathbf{F}^{\prime}$ assume a forma

$$
\mathbf{F}^{\prime}=\left(F_{1}^{\prime}, F_{2}^{\prime}, 0\right)
$$

onde

$$
\begin{aligned}
& F_{1}^{\prime}=F_{1} \cos 2 \alpha+F_{2} \sin 2 \alpha, F_{2}^{\prime}=F_{2} \cos 2 \alpha-F_{1} \sin 2 \alpha, \\
& F_{1}=F_{1}^{\prime} \cos 2 \alpha-F_{2}^{\prime} \sin 2 \alpha, F_{2}=F_{2}^{\prime} \cos 2 \alpha+F_{1}^{\prime} \sin 2 \alpha .
\end{aligned}
$$

Escolhendo $\mathbf{l}=(0,0,1)$ e selecionando $\alpha$ para ser uma solução da equação

$$
F_{1}=F_{1}^{\prime} \cos 2 \alpha, F_{2}=F_{1}^{\prime} \sin 2 \alpha, F_{3}=F_{3}^{\prime}+\dot{\alpha},
$$

obtemos

$$
\begin{aligned}
& F_{1}^{\prime}=F_{1} \cos 2 \alpha+F_{2} \sin 2 \alpha, F_{3}^{\prime}=F_{3}-\dot{\alpha}, \\
& F_{2}^{\prime}=F_{2} \cos 2 \alpha-F_{1} \sin 2 \alpha=0,
\end{aligned}
$$

e o campo externo reduzido $\mathbf{F}^{\prime}$ assume a forma

$$
\mathbf{F}^{\prime}=\left(F_{1}^{\prime}, 0, F_{3}^{\prime}\right) .
$$

Além disto, pode-se verificar que, se $V^{\prime}$ é uma solução da equação de spin com o campo externo (2.17), então: 
1. $U=(2)^{-1 / 2}\left(1+i \sigma_{1}\right) V^{\prime}$ é uma solução da equação de spin com o campo externo

$$
\mathbf{F}^{\prime}=\left(F_{1}^{\prime}, F_{3}^{\prime}, 0\right)
$$

2. $U=\sigma_{1} V^{\prime}$ é uma solução da equação de spin com o campo externo

$$
\mathbf{F}^{\prime}=\left(F_{1}^{\prime}, 0,-F_{3}^{\prime}\right)
$$

3. $U=\sigma_{3} V^{\prime}$ é uma solução da equação de spin com o campo externo

$$
\mathbf{F}^{\prime}=\left(-F_{1}^{\prime}, 0, F_{3}^{\prime}\right)
$$

4. $U=\sigma_{2} V^{\prime}$ é uma solução da equação de spin com o campo externo

$$
\mathbf{F}^{\prime}=\left(-F_{1}^{\prime}, 0,-F_{3}^{\prime}\right)
$$

5. $U=(2)^{-1 / 2}\left(\sigma_{1}+\sigma_{3}\right) V^{\prime}$ é uma solução da equação de spin com o campo externo

$$
\mathbf{F}^{\prime}=\left(F_{3}^{\prime}, 0, F_{1}^{\prime}\right)
$$

\subsection{Representação vetorial}

A seguinte relação é válida para quaisquer espinores $U, V$ e seus anticonjugados $\bar{U}, \bar{V}(2.5)$ :

$$
\begin{aligned}
& (U, \bar{V})=-(V, \bar{U}),(\bar{U}, V)=-(\bar{V}, U),(\bar{U}, \bar{V})=(V, U), \\
& (U, \bar{V})(\bar{V}, U)=(U, U)(V, V)-(U, V)(V, U)=(\bar{U}, V)(V, \bar{U}) \geq 0 .
\end{aligned}
$$

Dados dois espinores $U$ e $V$ podemos construir o vetor complexo $\mathbf{L}^{U, V}$ :

$$
\mathbf{L}^{U, V}=(U, \tilde{\sigma} V)=\left(u_{1}^{*} v_{2}+u_{2}^{*} v_{1}, i u_{2}^{*} v_{1}-i u_{1}^{*} v_{2}, u_{1}^{*} v_{1}-u_{2}^{*} v_{2}\right)
$$


o qual respeita as propriedades

$$
\begin{aligned}
& \text { i) }\left(\mathbf{L}^{U, V}\right)^{*}=\mathbf{L}^{V, U}, \mathbf{L}^{\bar{U}, \bar{V}}=-\mathbf{L}^{V, U}, \\
& \text { ii) } \mathbf{L}^{U, V} \mathbf{L}^{U^{\prime}, V^{\prime}}=2\left(U, V^{\prime}\right)\left(U^{\prime}, V\right)-(U, V)\left(U^{\prime}, V^{\prime}\right), \\
& \text { iii) } \mathbf{L}^{V, V} \mathbf{L}^{V, V}=(V, V)^{2}, \mathbf{L}^{\bar{V}, V} \mathbf{L}^{\bar{V}, V}=\mathbf{L}^{V, \bar{V}} \mathbf{L}^{V, \bar{V}}=0, \\
& \text { iv) } \mathbf{L}^{\bar{V}, V} \mathbf{L}^{V, \bar{V}}=2(V, V)^{2}, \mathbf{L}^{V, V} \mathbf{L}^{\bar{V}, V}=\mathbf{L}^{V, V} \mathbf{L}^{V, \bar{V}}=0, \\
& \text { v) }\left[\mathbf{L}^{V, \bar{V}} \times \mathbf{L}^{\bar{V}, V}\right]=2 i(V, V) \mathbf{L}^{V, V},\left[\mathbf{L}^{\bar{V}, V} \times \mathbf{L}^{V, V}\right]=i(V, V) \mathbf{L}^{\bar{V}, V}, \\
& \quad\left[\mathbf{L}^{V, V} \times \mathbf{L}^{V, \bar{V}}\right]=i(V, V) \mathbf{L}^{V, \bar{V}}, \\
& \text { vii) } \mathbf{L}^{U, V}=(V, V)^{-1}\left[(U, V) \mathbf{L}^{V, V}+(U, \bar{V}) \mathbf{L}^{\bar{V}, V}\right] .
\end{aligned}
$$

Usando a decomposição a seguir (2.8)

$$
U=(V, V)^{-1}[(V, U) V+(\bar{V}, U) \bar{V}]
$$

dado um vetor $\mathbf{p}$ e um espinor $V$ podemos obter,

$$
(\tilde{\sigma} \mathbf{p}) V=(V, V)^{-1}\left[\left(\mathbf{L}^{V, V} \mathbf{p}\right) V+\left(\mathbf{L}^{\bar{V}, V} \mathbf{p}\right) \bar{V}\right]
$$

As relações (2.25) implicam que todo espinor $V$ gera três vetores linearmente independentes,

$$
\mathbf{L}^{V, V}, \mathbf{L}^{\bar{V}, V}, \mathbf{L}^{V, \bar{V}}
$$

Todo vetor complexo a pode ser decomposto nestes vetores

$$
\begin{aligned}
& \mathbf{a}=a_{1} \mathbf{L}^{V, V}+a_{2} \mathbf{L}^{\bar{V}, V}+a_{3} \mathbf{L}^{V, \bar{V}}, \\
& a_{1}=\frac{\mathbf{a} \mathbf{L}^{V, V}}{(V, V)^{2}}, a_{2}=\frac{\mathbf{a} \mathbf{L}^{V, \bar{V}}}{2(V, V)^{2}}, a_{3}=\frac{\mathbf{a} \mathbf{L}^{\bar{V}, V}}{2(V, V)^{2}} .
\end{aligned}
$$

Com a ajuda dos vetores (2.27), podemos definir três vetores ortogonais reais

$$
\mathbf{e}_{1}=\frac{\mathbf{L}^{V, \bar{V}}+\mathbf{L}^{\bar{V}, V}}{2(V, V)}, \mathbf{e}_{2}=i \frac{\mathbf{L}^{V, \bar{V}}-\mathbf{L}^{\bar{V}, V}}{2(V, V)}, \mathbf{n}=\frac{\mathbf{L}^{V, V}}{(V, V)},
$$

estes obedecem às relações

$$
\mathbf{e}_{i} \mathbf{e}_{j}=\delta_{i j},\left[\mathbf{e}_{i} \times \mathbf{e}_{j}\right]=\epsilon_{i j k} \mathbf{e}_{k}, \mathbf{e}_{3}=\mathbf{n}
$$


onde $\epsilon_{i j k}$ é o símbolo de Levi-Civita $\left(\epsilon_{123}=1\right)$. As relações inversas têm a forma

$$
\mathbf{L}^{V, V}=(V, V) \mathbf{n}, \mathbf{L}^{\bar{V}, V}=(V, V)\left(\mathbf{e}_{1}+i \mathbf{e}_{2}\right), \mathbf{L}^{V, \bar{V}}=(V, V)\left(\mathbf{e}_{1}-i \mathbf{e}_{2}\right) .
$$

Um espinor $V$ sempre pode ser representado como

$$
V=N e^{i \frac{\alpha}{2}}\left(\begin{array}{c}
e^{-i \frac{\varphi}{2}} \cos \frac{\theta}{2} \\
e^{i \frac{\varphi}{2}} \sin \frac{\theta}{2}
\end{array}\right), N^{2}=(V, V)
$$

onde $N, \alpha, \theta$, e $\varphi$ são números reais. Nesta representação, o espinor anticonjugado tem a forma

$$
\bar{V}=N e^{-i \frac{\alpha}{2}}\left(\begin{array}{c}
-e^{-i \frac{\varphi}{2}} \sin \frac{\theta}{2} \\
e^{i \frac{\varphi}{2}} \cos \frac{\theta}{2}
\end{array}\right) .
$$

Considerando $\theta$ e $\varphi$ como ângulos de um sistema de referencial esférico, podemos definir os vetores unitários ortogonais $\mathbf{e}_{\varphi}, \mathbf{e}_{\theta}, \mathrm{e} \mathbf{n}$,

$$
\begin{aligned}
& \mathbf{e}_{\theta}=(\cos \theta \cos \varphi, \cos \theta \sin \varphi,-\sin \theta)=\left[\mathbf{e}_{\varphi} \times \mathbf{n}\right], \\
& \mathbf{e}_{\varphi}=(\sin \varphi, \cos \varphi, 0)=\left[\mathbf{n} \times \mathbf{e}_{\theta}\right], \\
& \mathbf{n}=(\sin \theta \cos \varphi, \sin \theta \sin \varphi, \cos \theta)=\left[\mathbf{e}_{\theta} \times \mathbf{e}_{\varphi}\right],
\end{aligned}
$$

em termo dos quais, os vetores (2.27) e (2.29) podem ser escritos como

$$
\begin{aligned}
& \mathbf{L}^{V, V}=N^{2} \mathbf{n}, \mathbf{L}^{\bar{V}, V}=N^{2}\left(\mathbf{e}_{\theta}+i \mathbf{e}_{\varphi}\right) e^{i \alpha}, \mathbf{L}^{V, \bar{V}}=N^{2}\left(\mathbf{e}_{\theta}-i \mathbf{e}_{\varphi}\right) e^{-i \alpha}, \\
& \mathbf{e}_{1}=\mathbf{e}_{\theta} \cos \alpha-\mathbf{e}_{\varphi} \sin \alpha, \mathbf{e}_{2}=\mathbf{e}_{\theta} \sin \alpha+\mathbf{e}_{\varphi} \cos \alpha
\end{aligned}
$$

Além disto, segue de (2.26) e (2.35) que

$$
(\tilde{\sigma} \mathbf{F}) V=(\mathbf{n F}) V+\left(\mathbf{F} \mathbf{e}_{\theta}+i \mathbf{F} \mathbf{e}_{\varphi}\right) \exp (i \alpha) \bar{V}
$$




\section{Capítulo 3}

\section{Equações relacionadas}

Como mencionado anteriormente, várias equações encontradas em diferentes problemas em física estão profundamente ligadas com a equação de spin. A seguir, vamos estabelecer estas ligações explicitando a relação entre estas equações. A ciência destas relações possibilita estender a aplicação do conhecimento das soluções de um determinado problema, além de permitir sua reformulação através de expressões mais convenientes. Como exemplo, em [16] a relação entre a equação de spin (1.3) e a equação de Schödinger na forma (3.3) abaixo é utilizada para obter as soluções (1.8), em [5] a relação entre (1.3) e um caso particular da equação de Euler (3.11) no final deste capítulo permite analisar uma série de propriedades de um feixe de moléculas numa cavidade sob a influência de um campo elétrico oscilante de amplitude constante.

\subsubsection{Equação de Schrödinger}

1. Considere a equação de Schrödinger em $0+1$ dimensão para um espinor $\Psi(t)$ com duas componentes complexas dependentes do tempo. No caso geral, esta equação assume a forma

$$
i \dot{\Psi}=H \Psi,
$$


onde o hamiltoniano $H$ é uma matriz $2 \times 2$ complexa dependente do tempo. A matriz $H$ pode sempre ser decomposta nas matrizes de base $H=F_{0} I+\tilde{\sigma} \mathbf{F}$, com $F_{0}=$ $F_{0}(t)$ e $\mathbf{F}=\left(F_{k}(t), k=1,2,3\right)$. Através da transformação $\Psi=V \exp \left(-i \int F_{0} d t\right)$, obtemos a equação de spin para o espinor $V$.

2. A equação de spin pode ser reduzida a um sistema de duas equações de Schrödinger unidimensionais independentes, em geral com potenciais complexos. Substituindo em (2.3) as funções

$$
v_{s}=\sqrt{A_{s}} \psi_{s}, A_{s}=F_{1}+(-1)^{s} i F_{2}
$$

obtemos as seguintes equações diferenciais lineares de segunda ordem

$$
\begin{aligned}
& \ddot{\psi}_{s}-V_{s} \psi_{s}=0, s=1,2 \\
& V_{s}=\frac{3}{4}\left(\frac{\dot{A}_{s}}{A_{s}}\right)^{2}-\frac{1}{2} \frac{\ddot{A}_{s}}{A_{s}}-A_{1} A_{2}-F_{3}^{2}-i(-1)^{s}\left(F_{3} \frac{\dot{A}_{s}}{A_{s}}-\dot{F}_{3}\right)
\end{aligned}
$$

Identificando as derivadas em (3.3) como derivadas espaciais $\left(\ddot{\psi}=d \psi / d x^{2}\right)$, cada uma destas equações pode ser interpretada com uma equação de Schrödinger estacionária unidimensional com potenciais complexos $V_{s}$.

\subsubsection{Equação de Euler}

Para um par de espinores $U$ e $V$ podemos definir o vetor complexo $\mathbf{L}^{U, V}=(U, \tilde{\sigma} V)$ (veja (2.24)), se $V$ é uma solução da equação de spin, então os vetores linearmente independentes $\mathbf{L}^{V, V}, \mathbf{L}^{\bar{V}, V}$ e $\mathbf{L}^{V, \bar{V}}(2.27)$ obedecem às seguintes equações

$$
\begin{aligned}
& \dot{\mathbf{L}}^{V, V}=i\left(\mathbf{F}^{*}-\mathbf{F}\right)(V, V)+\left[\left(\mathbf{F}+\mathbf{F}^{*}\right) \times \mathbf{L}^{V, V}\right], \\
& \dot{\mathbf{L}}^{\bar{V}, V}=2\left[\mathbf{F} \times \mathbf{L}^{\bar{V}, V}\right], \dot{\mathbf{L}}^{V, \bar{V}}=2\left[\mathbf{F}^{*} \times \mathbf{L}^{V, \bar{V}}\right],
\end{aligned}
$$

enquanto a equação de spin e as propriedades (2.25) implicam nas relações

$$
\mathbf{L}^{V, \dot{V}}=-i(V, V) \mathbf{F}+\left[\mathbf{F} \times \mathbf{L}^{V, V}\right], \mathbf{L}^{\bar{V}, \dot{V}}=\mathbf{L}^{\dot{\bar{V}}, V}=\left[\mathbf{F} \times \mathbf{L}^{\bar{V}, V}\right]
$$


Além disto, os vetores $\mathbf{e}_{1}, \mathbf{e}_{2}, \mathbf{n}(2.29)$,

$$
\mathbf{e}_{1}=\frac{\mathbf{L}^{V, \bar{V}}+\mathbf{L}^{\bar{V}, V}}{2(V, V)}, \mathbf{e}_{2}=i \frac{\mathbf{L}^{V, \bar{V}}-\mathbf{L}^{\bar{V}, V}}{2(V, V)}, \mathbf{n}=\frac{\mathbf{L}^{V, V}}{(V, V)},
$$

obedecem às seguintes equações:

$$
\begin{aligned}
& \dot{\mathbf{e}}_{1}=2 \mathbf{e}_{2}(\mathbf{K n})-2 \mathbf{n}\left(\mathbf{K} \mathbf{n}+\mathbf{G e}_{1}\right), \\
& \dot{\mathbf{e}}_{2}=2 \mathbf{n}\left(\mathbf{K} \mathbf{e}_{1}-\mathbf{G e}_{2}\right)-2 \mathbf{e}_{1}(\mathbf{K n}), \\
& \dot{\mathbf{n}}=2 \mathbf{e}_{1}\left(\mathbf{K e}_{2}+\mathbf{G e}_{1}\right)+2 \mathbf{e}_{2}\left(\mathbf{G e}_{2}-\mathbf{K} \mathbf{e}_{1}\right),
\end{aligned}
$$

com $\mathbf{K}$ e $\mathbf{G}$ dados por (1.4). Como $V$ obedece à equação de spin, através da representação $(2.32)$,

$$
V=N e^{i \frac{\alpha}{2}}\left(\begin{array}{c}
e^{-i \frac{\varphi}{2}} \cos \frac{\theta}{2} \\
e^{i \frac{\varphi}{2}} \sin \frac{\theta}{2}
\end{array}\right), N^{2}=(V, V),
$$

e dos vetores ortogonais (2.34),

$$
\begin{aligned}
& \mathbf{e}_{\theta}=\cos \theta(\cos \varphi, \sin \varphi,-\tan \theta)=\mathbf{e}_{1} \cos \alpha+\mathbf{e}_{2} \sin \alpha \\
& \mathbf{e}_{\varphi}=(\sin \varphi, \cos \varphi, 0)=\mathbf{e}_{2} \cos \alpha-\mathbf{e}_{1} \sin \alpha,
\end{aligned}
$$

podemos encontrar equações do movimento para os parâmetros $N, \alpha, \theta$, e $\varphi$. Tomando em conta a expressão para $\bar{V}(2.33)$ e a representação $(2.8)$ temos

$$
2 \dot{V}=\left(2 N^{-1} \dot{N}+i \dot{\alpha}-i \dot{\varphi} \cos \theta\right) V+(\dot{\theta}+i \dot{\varphi} \sin \theta) \exp (i \alpha) \bar{V}
$$

Finalmente, com o auxílio das expressões (3.8), (2.36) e (1.4) obtemos:

$$
\begin{aligned}
& \dot{\theta}=2 \mathbf{K e}_{\varphi}+2 \mathbf{G e}_{\theta}, \dot{\varphi} \sin \theta=2 \mathbf{G e}_{\varphi}-2 \mathbf{K e}_{\theta}, \\
& \dot{\alpha}=\dot{\varphi} \cos \theta-2 \mathbf{K n}, \dot{N}=N \mathbf{G n} .
\end{aligned}
$$

As equações (3.9) são autônomas, no sentido de não dependerem das funções $N$ e $\alpha$, e podem ser escritas na forma compacta

$$
\dot{\mathbf{n}}=2[\mathbf{G}-(\mathbf{G n}) \mathbf{n}]+2[\mathbf{K} \times \mathbf{n}] .
$$


Assim, a evolução temporal do vetor $\mathbf{n}$ é determinada apenas pelo campo externo. A integração do sistema (3.11) permite obter $\theta(t)$ e $\varphi(t)$. Com isto, de (3.10), temos

$$
\alpha=\int(\dot{\varphi} \cos \theta-2 \mathbf{K n}) d t, N=\exp \int \mathbf{G n} d t
$$

A equação (3.11) para $\mathbf{G}=0$ é a conhecida equação de Euler. Ela aparece, por exemplo, na teoria do giroscópio, na teoria de um giromagneto clássico em um campo magnético, na teoria da ressonância eletromagnética (veja [5]). Para $\mathbf{G} \neq 0$ esta equação pode ser usada para descrever um sistema amortecido. 


\section{Capítulo 4}

\section{Sobre as soluções da equação de spin}

\subsection{Solução geral da equação de spin}

A solução geral da equação de spin pode ser escrita como

$$
Y_{\text {ger }}(t)=a V(t)+b U(t),
$$

onde $a$ e $b$ são constantes complexas arbitrárias, enquanto $V(t)$ e $U(t)$ são duas soluções particulares, linearmente independentes, da equação de spin. Na verdade, apenas uma solução $V$ precisa ser conhecida, uma vez que outra solução $U$, linearmente independente, pode ser construída a partir desta e de seu anticonjugado (2.5), através da relação (2.8)

$$
U(t)=\alpha(t) V(t)+\beta(t) \bar{V}(t),
$$

onde $\alpha(t)$ e $\beta(t)$ são funções complexas do tempo. Substituindo a expressão (4.2) na equação de spin (1.3), e levando em conta (2.6) e (1.4) temos

$$
\dot{\alpha} V+\dot{\beta} \bar{V}=2 \beta \tilde{\sigma} \mathbf{G} \bar{V} .
$$

Portanto, utilizando o vetor $\mathbf{L}^{U, V}(2.24)$ e as relações (2.25), podemos multiplicar a equação acima por $V^{+}$e $\bar{V}^{+}$para obter

$$
(V, V) \dot{\beta}=-2 \beta \mathbf{L}^{V, V} \mathbf{G}, \dot{\alpha}=2 \beta(V, V)^{-1} \mathbf{L}^{V, \bar{V}} \mathbf{G} .
$$


Com a ajuda das expressões (2.35) e (3.10) a primeira destas equações pode ser escrita como

$$
N \dot{\beta}=-2 \dot{N} \beta,
$$

a qual pode ser facilmente integrada

$$
\beta=\beta_{0} N^{-2}=\beta_{0}(V, V)^{-1},
$$

onde $\beta_{0}$ é uma constante complexa arbitrária. Com isto, a segunda equação em (4.4) implica

$$
\dot{\alpha}=2 \beta_{0}(V, V)^{-2} \mathbf{L}^{V, \bar{V}} \mathbf{G},
$$

de forma que $\alpha(t)$ pode ser encontrada por integração

$$
\alpha=\alpha_{0}+2 \beta_{0} \int(V, V)^{-2} \mathbf{L}^{V, \bar{V}} \mathbf{G} d t
$$

onde $\alpha_{0}$ é uma constante complexa arbitrária. Concluindo, a solução geral $Y_{\text {ger }}$ da equação de spin, pode ser construída a partir de uma solução particular $V$, fazendo

$$
Y_{\text {ger }}=\left[\alpha_{0}+2 \beta_{0} \int(V, V)^{-2} \mathbf{L}^{V, \bar{V}} \mathbf{G} d t\right] V+\beta_{0}(V, V)^{-1} \bar{V},
$$

com $\alpha_{0}$ e $\beta_{0}$ constantes complexas arbitrárias.

\subsection{Soluções estacionárias}

Considere a equação de spin com um campo magnético externo constante, F. Neste caso, podemos procurar por soluções estacionárias na forma

$$
V(t)=\exp (-i \lambda t) V
$$

onde $V$ é um espinor independente do tempo que respeita a equação

$$
(\tilde{\sigma} \mathbf{F}) V=\lambda V
$$


Uma análise mais detalhada da relação acima é apresentada no apêndice. Em particular, para $\mathbf{F}^{2} \neq 0$, temos duas soluções independentes $V_{\zeta}, \lambda_{\zeta}, \zeta= \pm 1$,

$$
\begin{aligned}
& V_{1}=N_{1}\left(\begin{array}{c}
F_{3}+\sqrt{\mathbf{F}^{2}} \\
i F_{2}+F_{1}
\end{array}\right), \lambda_{1}=\sqrt{\mathbf{F}^{2}}, \\
& V_{-1}=N_{-1}\left(\begin{array}{c}
i F_{2}-F_{1} \\
F_{3}+\sqrt{\mathbf{F}^{2}}
\end{array}\right), \lambda_{-1}=-\sqrt{\mathbf{F}^{2}} .
\end{aligned}
$$

onde $N_{\zeta}$ é um fator de normalização.

\subsection{A matriz de transformação}

Existem conjuntos de campos externos que geram equações de spin equivalentes, no sentido de suas soluções estarem ligadas por certas transformações inversíveis. Suponha o conhecimento de uma solução $V_{1}$ da equação de spin com o campo externo $\mathbf{F}_{1}$,

$$
i \dot{V}_{1}=\left(\tilde{\sigma} \mathbf{F}_{1}\right) V_{1},
$$

e que se deseja encontrar uma matriz não singular dependente do tempo $\hat{T}^{21}$, a qual chamaremos matriz de transformação, de forma que o espinor $V_{2}$,

$$
V_{2}(t)=\hat{T}^{21}(t) V_{1}(t)
$$

seja solução da equação de spin para um determinado campo externo $\mathbf{F}_{2}$,

$$
i \dot{V}_{2}=\left(\tilde{\sigma} \mathbf{F}_{2}\right) V_{2} .
$$

Substituindo (4.13) em (4.14), e usando a equação de spin, temos que a matriz de transformação respeita a relação

$$
i \frac{d}{d t} \hat{T}^{21}=\left(\tilde{\sigma} \mathbf{F}_{2}\right) \hat{T}^{21}-\hat{T}^{21}\left(\tilde{\sigma} \mathbf{F}_{1}\right) .
$$

Como toda matriz $2 \times 2$, a matriz $\hat{T}^{21}$ pode ser escrita como

$$
\hat{T}^{21}=a_{0}-i \tilde{\sigma} \mathbf{a}, \mathbf{a}=\left(a_{1}, a_{2}, a_{3}\right),
$$


onde $a_{s}(t), s=0,1,2,3$ são funções complexas dependentes do tempo. Substituindo (4.16) em (4.15), e usando propriedades elementares das matrizes de Pauli, obtém-se o seguinte conjunto de equações para as funções $a_{s}$ :

$$
\begin{aligned}
& \dot{a}_{0}+\mathbf{a} \mathbf{F}_{21}=0, \mathbf{F}_{21}=\mathbf{F}_{2}-\mathbf{F}_{1}, \\
& \dot{\mathbf{a}}+2\left[\mathbf{a} \times \mathbf{F}_{1}\right]+\left[\mathbf{a} \times \mathbf{F}_{21}\right]-a_{0} \mathbf{F}_{21}=0 .
\end{aligned}
$$

É fácil verificar que $\Delta=\operatorname{det} \hat{T}^{21}=a_{0}^{2}+\mathbf{a}^{2}$ é uma integral do movimento. Sendo a matriz $\hat{T}^{21}$ determinada por (4.15), a menos de uma constante multiplicativa, podemos escolher, sem perda de generalidade,

$$
\Delta=a_{0}^{2}+\mathbf{a}^{2}=1
$$

Para a matriz inversa $\left(\hat{T}^{21}\right)^{-1}$ temos

$$
\left(\hat{T}^{21}\right)^{-1}=\Delta^{-1}\left(a_{0}+i \tilde{\sigma} \mathbf{a}\right)=a_{0}+i \tilde{\sigma} \mathbf{a} .
$$

Para dois campos $\mathbf{F}_{1}$ e $\mathbf{F}_{2}$ dados, as equações (4.17) formam um sistema linear homogêneo (complexo) de quatro equações diferenciais ordinárias de primeira ordem. Resolver este sistema é completamente equivalente a resolver a equação de spin com um campo externo $\mathbf{F}_{2}$. Além disto, assumindo como conhecidos o campo externo $\mathbf{F}_{1}$ e a matriz $\hat{T}^{21}$, podemos de (4.17) obter o campo externo $\mathbf{F}_{2}$, entretanto, para realizar este desenvolvimento, precisamos considerar dois casos:

1. Para $a_{0} \neq 0$, introduzimos o vetor complexo $\mathbf{q}=\mathbf{q}(t)$,

$$
\mathbf{q}=\mathbf{a} / a_{0}
$$

tal que $\mathbf{q}^{2} \neq-1$. Com isto, (4.18) implica em

$$
a_{0}=\left(1+\mathbf{q}^{2}\right)^{-1 / 2}
$$

De (4.17) obtemos a equação

$$
\dot{\mathbf{q}}-\mathbf{q}\left(\mathbf{q} \mathbf{F}_{21}\right)+\left[\mathbf{q} \times \mathbf{F}_{21}\right]+2\left[\mathbf{q} \times \mathbf{F}_{1}\right]-\mathbf{F}_{21}=0 .
$$


Esta equação permite obter uma representação única para o campo externo $\mathbf{F}_{2}$,

$$
\mathbf{F}_{2}=\frac{\dot{\mathbf{q}}+[\mathbf{q} \times \dot{\mathbf{q}}]+2\left[\mathbf{q} \times \mathbf{F}_{1}\right]+2 \mathbf{q}\left(\mathbf{q} \mathbf{F}_{1}\right)-2 \mathbf{q}^{2} \mathbf{F}_{1}}{1+\mathbf{q}^{2}}+\mathbf{F}_{1} .
$$

Neste caso, a matriz de transformação é dada por

$$
\hat{T}^{21}=\frac{1-i \tilde{\sigma} \mathbf{q}}{\sqrt{1+\mathbf{q}^{2}}},\left(\hat{T}^{21}\right)^{-1}=\frac{1+i \tilde{\sigma} \mathbf{q}}{\sqrt{1+\mathbf{q}^{2}}} .
$$

2. Para $a_{0}=0$, introduzimos o vetor complexo $\mathbf{q}=\mathbf{q}(t)$,

$$
\mathbf{q}=-i \mathbf{a}
$$

Neste caso, a condição (4.18) implica $\mathbf{q}^{2}=-1$ e obtemos de (4.17)

$$
\dot{\mathbf{q}}+\left[\mathbf{q} \times \mathbf{F}_{21}\right]+2\left[\mathbf{q} \times \mathbf{F}_{1}\right]=0, \mathbf{q F}_{21}=0 .
$$

De (4.25) construímos, de forma única, o vetor $\mathbf{F}_{2}$,

$$
\mathbf{F}_{2}=[\mathbf{q} \times \dot{\mathbf{q}}]+2 \mathbf{q}\left(\mathbf{q F}_{1}\right)-\mathbf{F}_{1}
$$

A matriz de transformação agora é dada por

$$
\hat{T}^{21}=\left(T^{21}\right)^{-1}=\tilde{\sigma} \mathbf{q} .
$$

Conseqüentemente, dada uma solução exata $V_{1}$, correspondente a um campo externo $\mathbf{F}_{1}$, podemos construir uma família de campos externos $\mathbf{F}_{2}$, e suas correspondentes soluções $V_{2}$ (4.13), parametrizados por um vetor complexo arbitrário dependente do tempo $\mathbf{q}(t)$. Para o caso de campos $\mathbf{F}_{1}$ reais, podemos construir novos campos $\mathbf{F}_{2}$, também reais, assumindo q um vetor real. Neste caso, a matriz de transformação é unitária.

\subsection{O operador de evolução}

Vamos continuar o desenvolvimento anterior denotando $\mathbf{F}_{2}=\mathbf{F}$ e $\hat{T}^{21}=\hat{T}$. Além disto, vamos escolher $\mathbf{F}_{1}=0$ e $V_{1}$ um espinor constante arbitrário, $V_{1}=$ const. Com isto, a matriz de transformação obedecerá à equação (4.15)

$$
i \frac{d \hat{T}}{d t}=(\tilde{\sigma} \mathbf{F}) \hat{T} .
$$


Se a matriz de transformação $\hat{T}$ é conhecida, o operador de evolução $\hat{R}$, o qual representa uma solução de (4.28) com condição inicial $\hat{R}(0)=I$, pode ser construído como

$$
\hat{R}(t)=\hat{T}(t) \hat{T}^{-1}(0) .
$$

Como apresentado a seguir, a relação acima e o resultado da seção anterior permitem construir o operador de evolução da equação de spin a partir das expressões obtidas para a matriz de transformação.

1. Vamos selecionar um vetor complexo arbitrário dependente do tempo $\mathbf{q}(t)(\mathbf{q}(0)=$ $\left.\mathbf{q}_{0}\right)$, tal que $\mathbf{q}^{2} \neq-1$. Então, a equação de spin com o campo externo

$$
\mathbf{F}=\frac{\dot{\mathbf{q}}+[\mathbf{q} \times \dot{\mathbf{q}}]}{1+\mathbf{q}^{2}},
$$

tem como operador de evolução

$$
\hat{R}=\frac{(1-i \tilde{\sigma} \mathbf{q})\left(1+i \tilde{\sigma} \mathbf{q}_{0}\right)}{\sqrt{\left(1+\mathbf{q}^{2}\right)\left(1+\mathbf{q}_{0}^{2}\right)}}=\frac{1+\mathbf{q q}_{0}-i \tilde{\sigma} \mathbf{p}}{\sqrt{\left(1+\mathbf{q}^{2}\right)\left(1+\mathbf{q}_{0}^{2}\right)}},
$$

onde $\mathbf{p}=\mathbf{q}-\mathbf{q}_{0}+\left[\mathbf{q}_{0} \times \mathbf{q}\right]$.

2. Para o vetor complexo $\mathbf{q}(t)\left(\mathbf{q}(0)=\mathbf{q}_{0}\right)$ escolhido com $\mathbf{q}^{2}=-1$, a solução da equação de spin com o campo externo

$$
\mathbf{F}=[\mathbf{q} \times \dot{\mathbf{q}}],
$$

tem como operador de evolução

$$
\hat{R}=(\tilde{\sigma} \mathbf{q})\left(\tilde{\sigma} \mathbf{q}_{0}\right)=\mathbf{q} \mathbf{q}_{0}+i \tilde{\sigma}\left[\mathbf{q} \times \mathbf{q}_{0}\right]
$$

Para campos externos reais q é um vetor real e $\hat{R}$ um operador unitário. 


\section{Capítulo 5}

\section{O problema inverso para a equação de spin}

Dado um espinor arbitrário, o problema inverso consiste em determinar se existe um campo externo $\mathbf{F}$ para o qual este espinor seja uma solução da equação de spin.

Para um espinor qualquer $V$, podemos construir os três vetores linearmente independentes $\mathbf{L}^{V, V}, \mathbf{L}^{V, \bar{V}}, \mathbf{L}^{\bar{V}, V}$ (veja (2.27)), e decompor o campo externo $\mathbf{F}$ nestes vetores

$$
\mathbf{F}=c_{1} \mathbf{L}^{V, V}+c_{2} \mathbf{L}^{V, \bar{V}}+c \mathbf{L}^{\bar{V}, V}
$$

onde $c_{1}, c_{2}$, e $c$ são coeficientes dependentes do tempo. Substituindo esta expressão na equação de spin e usando as fórmulas (2.8) e (2.26), encontramos

$$
i \dot{V}=(\tilde{\sigma} \mathbf{F}) V=(V, V)\left(c_{1} V+2 c_{2} \bar{V}\right)
$$

Multiplicando esta relação pela esquerda por $V^{+}$e $\bar{V}^{+}$, obtemos

$$
c_{1}=i \frac{(V, \dot{V})}{(V, V)^{2}}, c_{2}=i \frac{(\bar{V}, \dot{V})}{2(V, V)^{2}} .
$$

Substituindo (5.3) em (5.1), temos

$$
\mathbf{F}=\frac{i}{2(V, V)^{2}}\left[2(V, \dot{V}) \mathbf{L}^{V, V}+(\bar{V}, \dot{V}) \mathbf{L}^{V, \bar{V}}\right]+c \mathbf{L}^{\bar{V}, V}
$$


onde a função complexa $c(t)$ permanece completamente arbitrária. Portanto, existe uma infinidade de campos externos $\mathbf{F}$, que admitem a mesma solução $V$ da equação de spin e esta arbitrariedade funcional está completamente descrita em (5.4).

Usando (2.28), a equação (5.4) pode ser reescrita como

$$
\mathbf{F}=\frac{i}{2(V, V)^{2}}\left\{(V, V)\left(\mathbf{L}^{V, \dot{V}}-\mathbf{L}^{\dot{V}, V}\right)+[(V, \dot{V})+(\dot{V}, V)] \mathbf{L}^{V, V}\right\}+b \mathbf{L}^{\bar{V}, V},
$$

com $b(t)$ uma nova função complexa arbitrária. A arbitrariedade funcional presente na solução do problema inverso está relacionada com o fato do espinor $V$ ser dado apenas por duas funções complexas, enquanto o campo externo $\mathbf{F}$ é definido por três funções complexas.

Desta forma, mostramos que todo o espinor complexo com uma dependência temporal arbitrária, desde que diferenciável, é solução de uma certa família de equações de spin.

Levando em conta a seguinte forma explícita do espinor (2.32),

$$
V=N e^{i \frac{\alpha}{2}}\left(\begin{array}{c}
e^{-i \frac{\varphi}{2}} \cos \frac{\theta}{2} \\
e^{i \frac{\varphi}{2}} \sin \frac{\theta}{2}
\end{array}\right), N^{2}=(V, V)
$$

e usando as fórmulas (2.35) e (3.8), pode-se facilmente obter de (5.4) a expressão

$$
\mathbf{F}=\frac{1}{2}\left[(\dot{\varphi} \cos \theta-\dot{\alpha}) \mathbf{n}-\dot{\varphi} \mathbf{e}_{\theta} \sin \theta+\dot{\theta} \mathbf{e}_{\varphi}\right]+i \frac{\dot{N}}{N} \mathbf{n}+a\left(\mathbf{e}_{\theta}+i \mathbf{e}_{\varphi}\right),
$$

onde $a(t)$ é uma função complexa arbitrária. 


\section{Capítulo 6}

\section{Equação de spin simétrica}

Chamaremos equação de spin simétrica o caso em que $\mathbf{F}$ é um campo externo real. Neste caso, de acordo com (1.4), temos

$$
\mathbf{F}=\operatorname{Re} \mathbf{F}=\mathbf{K}, \operatorname{Im} \mathbf{F}=\mathbf{G}=0 .
$$

Com isto, a equação de spin assume a forma de uma equação de Schrödinger com um hamiltoniano hermitiano $H$,

$$
i \dot{V}=H V, H=\tilde{\sigma} \mathbf{F}=H^{+} .
$$

Entretanto, mesmo para a equação de spin simétrica, o hamiltoniano unidimensional (3.3) não é hermitiano no caso geral.

O caso simétrico da equação de spin apresenta uma série de propriedades, as quais, em geral, não estão presentes na equação de spin para um campo externo complexo. Por exemplo, a unitariedade da matriz de transformação (4.13) e do operador de evolução (4.29).

Vale notar que, para a equação de spin simétrica, o desenvolvimento da matriz de transformação (4.16) continua válido $^{1}$, desde que o vetor q em (4.24) e (4.27) seja real.

\footnotetext{
${ }^{1} \mathrm{Ou}$ seja, o novo campo externo obtido a partir da matriz de transformação fornece uma nova equação de spin simétrica.
} 
Além disto, para as equações relacionadas à equação de spin (capítulo 3), no caso simétrico, algumas propriedades adicionais podem ser estabelecidas. Por exemplo, as equações de evolução (3.5) para os vetores linearmente independentes (2.27) tornam-se coincidentes, de sorte que estes vetores devem ser distinguidos por uma escolha apropriada das condições iniciais.

A seguir, apresentaremos outras características peculiares à equação de spin simétrica.

\subsection{A solução geral e o problema inverso}

A solução geral $Y_{\text {gen }}$ para a equação de spin simétrica tem a forma

$$
Y_{\text {gen }}=a V+b \bar{V}
$$

onde $V$ é uma solução particular, não nula, da equação de spin e $a, b$ constantes complexas arbitrárias. Este resultado provém de (4.4), pois $\mathbf{G}=\operatorname{Im} \mathbf{F}=0$.

Para qualquer solução $V$ da equação de spin simétrica, a quantidade $N^{2}=(V, V)$ se conserva no tempo, o que pode ser visto de (3.10) para o caso em que $\mathbf{G}=0$. Entretanto, o inverso não é sempre verdade. O fato de $N^{2}$ ser constante, não implica que $V$ seja uma solução da equação de spin simétrica, uma vez que, de acordo com (5.4), pode-se obter toda uma família de campos externos complexos que admitem soluções com este $N^{2}=$ const.

Para um espinor $V$ diferenciável arbitrário, não nulo e sujeito à condição $(V, V)=$ const, existe apenas uma equação de spin simétrica, i.e., apenas um campo externo real, cuja solução é dada por este espinor e a solução geral tem a forma (6.3), isto segue de (5.5). Neste caso, um campo externo real pode ser construído a partir de $V$ de forma única

$$
\mathbf{F}=i[2(V, V)]^{-1}\left(\mathbf{L}^{V, \dot{V}}-\mathbf{L}^{\dot{V}, V}\right)
$$

Pode ser verificado facilmente que a mesma expressão para $\mathbf{F}$ surge quando $V$ é substituído por $Y_{\text {gen }}(6.3)$, o que confirma a unicidade do campo externo F. Apresentando $V$ na forma 
(2.32), e fazendo $N=$ const, obtém-se a decomposição de $\mathbf{F}$ nos vetores de base do sistema de coordenadas esférico (2.34):

$$
\mathbf{F}=\frac{1}{2}\left[(\dot{\varphi} \cos \theta-\dot{\alpha}) \mathbf{n}-\dot{\varphi} \mathbf{e}_{\theta} \sin \theta+\dot{\theta} \mathbf{e}_{\varphi}\right]
$$

Com isto, pode-se encontrar as componentes cartesianas do campo externo $\mathbf{F}$ e calcular seu quadrado,

$$
\begin{aligned}
& \mathbf{F}=\frac{1}{2}(-\dot{\theta} \sin \varphi-\dot{\alpha} \sin \theta \cos \varphi, \dot{\theta} \cos \varphi-\dot{\alpha} \sin \theta \sin \varphi, \dot{\varphi}-\dot{\alpha} \cos \theta), \\
& F^{2}=\mathbf{F}^{2}=\frac{1}{4}\left(\dot{\theta}^{2}+\dot{\varphi}^{2}+\dot{\alpha}^{2}-2 \dot{\alpha} \dot{\varphi} \cos \theta\right) .
\end{aligned}
$$

A possibilidade de uma construção única do campo externo real $\mathbf{F}$, para um espinor arbitrário $V(t)$ com norma constante implica também na possibilidade de gerar equações de spin simétricas e suas soluções exatas.

\subsection{Formas lagrangiana e hamiltoniana da equação de spin simétrica}

Considere o sistema de equações (3.9) para um campo externo real. Neste caso, o sistema pode ser escrito como

$$
\dot{\theta}=2\left(F_{2} \cos \varphi-F_{1} \sin \varphi\right), \dot{\varphi} \sin \theta=2 F_{3} \sin \theta-2\left(F_{1} \cos \varphi+F_{2} \sin \varphi\right) \cos \theta
$$

Sem perda de generalidade, pode-se sempre escolher

$$
F_{1}=g(t) \cos 2 \alpha(t), F_{2}=g(t) \sin 2 \alpha(t)
$$

onde $g(t)$ e $\alpha(t)$ são funções reais dependentes do tempo.Vamos substituir $\varphi(t)$ na equação (6.7) por uma nova função $\Phi(t)$ dada por

$$
\varphi(t)=\Phi(t)+2 \alpha(t)
$$


Com isto, o sistema (6.7) assume a forma

$$
\dot{\theta}=-2 g \sin \Phi, \dot{\Phi} \sin \theta=2 f \sin \theta-2 g \cos \Phi \cos \theta
$$

onde

$$
f(t)=F_{3}(t)-\dot{\alpha}(t)
$$

Note que a substituição (6.9) é equivalente à transformação (2.11), para uma escolha de $\mathbf{e}=(0,0,1)$ e selecionando $\alpha(t)$, tal que o campo externo assuma a forma (2.17).

Agora, a introdução da coordenada $q$, do momento conjugado $p$ e do hamiltoniano $H$,

$$
q=\cos \theta, p=-\Phi, H=2 g \sqrt{1-q^{2}} \cos p+2 q f,
$$

faz com que o sistema (6.10) assuma a forma das equações de Hamilton unidimensionais $[5,16]$

$$
\dot{q}=\frac{\partial H}{\partial p}, \dot{p}=-\frac{\partial H}{\partial q} .
$$

Realizando transformações canônicas, é possível obter diferentes formas das equações de Hamilton associadas à equação de spin simétrica.

As equações (6.10) podem também ser obtidas através das equações de Euler-Lagrange para a função lagrangiana

$$
\mathcal{L}=[(1-\gamma) \dot{\theta} \Phi-2 g \cos \Phi] \sin \theta+[\gamma \dot{\Phi}-2 f] \cos \theta
$$

onde $\gamma$ é um número real arbitrário.

Finalmente, o conjunto (6.10) implica uma equação diferencial de segunda ordem para a função $\theta(t)$,

$$
\ddot{\theta}-\frac{\dot{g}}{g} \dot{\theta}+2 f \sqrt{4 g^{2}-\dot{\theta}^{2}}-\left(4 g^{2}-\dot{\theta}^{2}\right) \frac{\cos \theta}{\sin \theta}=0,
$$

a qual pode também ser obtida pelas equações de Euler-Lagrange através da lagrangiana

$$
\mathcal{L}=\dot{\theta} \arcsin (\dot{\theta} / 2 g) \sin \theta+\sqrt{4 g^{2}-\dot{\theta}^{2}} \sin \theta+2 f \cos \theta .
$$

A lagrangiana acima implica na seguinte hamiltoniana

$$
H=-2\left[g \cos \left(\frac{p}{\sin \theta}\right) \sin \theta+f \cos \theta\right], p=\arcsin (\dot{\theta} / 2 g) \sin \theta
$$

a qual, pelas equações canônicas (6.13), fornece a equação (6.15). 


\section{Capítulo 7}

\section{Soluções exatas da equação de spin}

No capítulo 3 apresentamos a relação entre a equação de spin e outros problemas em física. Estas relações permitem utilizar soluções conhecidas destes problemas para encontrar novas soluções da equação de spin. Neste capítulo vamos apresentar explicitamente uma série destas soluções construídas a partir das soluções exatas da equação de Schrödinger estacionária unidimensional encontradas em [23]. Entretanto, antes de prosseguirmos, são necessárias três observações:

1. Seja $V$ uma solução da equação de spin para um certo campo externo F. Nesta equação, realizamos a seguinte transformação

$$
t=T\left(t^{\prime}\right)
$$

onde $T\left(t^{\prime}\right)$ é uma função inversível real do novo parâmetro real $t^{\prime}$. Então, a equação de spin toma a forma

$$
i \frac{d V^{\prime}(t)}{d t}=\left(\tilde{\sigma} \mathbf{F}^{\prime}(t)\right) V^{\prime}(t),
$$

onde

$$
\mathbf{F}^{\prime}(t)=\mathbf{F}(T(t)) \dot{T}, V^{\prime}(t)=V(T(t)) .
$$

Conseqüentemente, o conhecimento da solução $V$, com campo externo $\mathbf{F}$, permite construir soluções $V^{\prime}$ para uma família de campos externos $\mathbf{F}^{\prime}$, parametrizados pe- 
las funções $T$. Dizemos que todas as funções relacionadas por este tipo de reparametrização pertencem à mesma classe de equivalência. Todas as soluções apresentadas aqui pertencem a diferentes classes de equivalência.

2. Como vimos anteriormente, uma equação de spin com um campo externo arbitrário pode ser reduzida a uma equação de spin equivalente com um campo externo na forma (2.17), o qual possui apenas duas componentes não nulas

$$
\mathbf{F}=\left(F_{1}, 0, F_{3}\right)
$$

Todas as soluções listadas aqui representam soluções para campos externos na forma acima.

3. Para campos externos na forma (7.4), cujas componentes $F_{1}$ e $F_{3}$ sejam proporcionais, podemos, sem perda de generalidade, escrever

$$
F_{1}=q \sin \lambda, F_{3}=q \cos \lambda,
$$

onde $q(t)$ é uma certa função do tempo e $\lambda$ uma constante complexa. Seja $\omega(t)$ uma função definida pela relação

$$
\dot{\omega}=q, \omega(0)=0 .
$$

Então, o operador de evolução para a equação de spin com o campo externo (7.5) tem a forma

$$
\hat{R}=\cos \omega-i\left(\sigma_{1} \sin \lambda+\sigma_{3} \cos \lambda\right) \sin \omega t .
$$

Consideraremos campos externos com componentes $F_{1}$ e $F_{3}$ não nulas e linearmente independentes. Como pode ser verificado, tivemos sucesso na obtenção de 26 pares destas funções. A seguir, apresentaremos estas funções, bem como os espinores $V$ que correspondem às soluções exatas para as equações de spin correspondentes. Nestas expressões, a seguinte notação será usada (em acordo com [22]):

$F(\alpha, \beta, \gamma, z)$ são as funções hipergeométricas de Gauss; 
$\Phi(\alpha, \gamma, z)$ são as funções hipergeométricas degeneradas;

$D_{p}(z)$ são as funções cilíndricas parabólicas;

$\varphi=\omega t+\varphi_{0}$

$\omega$ e $\varphi_{0}$ são constantes reais;

$a, b, c$ e $\alpha, \beta, \gamma, \lambda, \mu, \nu$ são constantes complexas.

\subsection{Lista das soluções exatas}

1. $F_{1}=a t, F_{3}=b t+c / t$ :

$$
\begin{array}{r}
V=\left(\begin{array}{c}
a t^{\gamma+2} e^{-z / 2} \Phi(\alpha+1, \gamma+2 ; z) \\
2(i-c) t^{\gamma} e^{-z / 2} \Phi(\alpha, \gamma ; z)
\end{array}\right), \\
z=i t^{2} \sqrt{a^{2}+b^{2}}, \alpha=\frac{\gamma}{2}\left(1+\frac{b}{\sqrt{a^{2}+b^{2}}}\right), \gamma=i c .
\end{array}
$$

2. $F_{1}=a / t, F_{3}=b / t+c t$ :

$$
\begin{gathered}
V=\left(\begin{array}{l}
-a t^{\gamma-1} e^{-z / 2} \Phi(\alpha, \gamma ; z) \\
\left(\sqrt{a^{2}+b^{2}}+b\right) t^{\gamma-1} e^{-z / 2} \Phi(\alpha+1, \gamma ; z)
\end{array}\right), \\
z=i c t^{2}, 2 \alpha=i\left(\sqrt{a^{2}+b^{2}}+b\right), \gamma=1+i \sqrt{a^{2}+b^{2}} .
\end{gathered}
$$

3. $F_{1}=a / t, F_{3}=b / t+c:$

$$
\begin{gathered}
V=\left(\begin{array}{c}
-a t^{(\gamma-1) / 2} e^{-z / 2} \Phi(\alpha, \gamma ; z) \\
-i a t^{(\gamma-1) / 2} e^{-z / 2} \Phi(1+\alpha, \gamma ; z)
\end{array}\right), \\
z=2 i c t, \alpha=i\left(\sqrt{a^{2}+b^{2}}+b\right), \gamma=1+2 i \sqrt{a^{2}+b^{2}} .
\end{gathered}
$$

4. $F_{1}=a / \sin 2 \varphi, F_{3}=(b \cos 2 \varphi+c) / \sin 2 \varphi$ :

$$
\begin{gathered}
V=\left(\begin{array}{l}
-a z^{\mu}(1-z)^{\nu} F(\alpha+1, \beta ; \gamma ; z) \\
(-4 i \omega \mu+b+c) z^{\mu}(1-z)^{\nu} F(\alpha, \beta+1 ; \gamma ; z)
\end{array}\right), \\
z=\sin ^{2} \varphi, \mu=\frac{i}{4 \omega} \sqrt{a^{2}+(b+c)^{2}}, \nu=\frac{i}{4 \omega} \sqrt{a^{2}+(b-c)^{2}}, \\
\alpha=\mu+\nu-i b / 2 \omega, \beta=\mu+\nu+i b / 2 \omega, \gamma=1+2 \mu .
\end{gathered}
$$


5. $F_{1}=a \tan \varphi, F_{3}=b \tan \varphi+c \cot \varphi$ :

$$
\begin{array}{r}
V=\left(\begin{array}{c}
2(c+i \omega) z^{\mu}(1-z)^{\nu} F(\alpha, \beta ; 2 \mu ; z) \\
a z^{\mu+1}(1-z)^{\nu} F(\alpha+1, \beta+1 ; 2 \mu+2 ; z)
\end{array}\right), \\
z=\sin ^{2} \varphi, \mu=-\frac{i c}{2 \omega}, \nu=\frac{i}{2 \omega} \sqrt{a^{2}+b^{2}}, \\
\lambda=\frac{i}{2 \omega} \sqrt{a^{2}+(b-c)^{2}}, \alpha=\nu+\mu+\lambda, \beta=\nu+\mu-\lambda .
\end{array}
$$

6. $F_{1}=a / \sin \varphi, F_{3}=b \tan \varphi+c \cot \varphi$ :

$$
\begin{array}{r}
V=\left(\begin{array}{c}
-a z^{\mu}(1-z)^{\nu+1 / 2} F(\alpha+1, \beta ; 2 \mu+1 ; z) \\
\left(\sqrt{a^{2}+c^{2}}+c\right) z^{\mu}(1-z)^{\nu} F(\alpha, \beta ; 2 \mu+1 ; z)
\end{array}\right), \\
\mu=\frac{i}{2 \omega} \sqrt{a^{2}+c^{2}}, \nu=-\frac{i b}{2 \omega}, z=\sin ^{2} \varphi, \\
\alpha=\mu-\frac{i c}{2 \omega}, \beta=\frac{1}{2}+\mu+2 \nu+\frac{i c}{2 \omega} .
\end{array}
$$

7. $F_{1}=a / \cos \varphi, F_{3}=b \tan \varphi+c$ :

$$
\begin{array}{r}
V=\left(\begin{array}{c}
(\omega+2 c-2 i b) z^{\mu}(1-z)^{\nu} F(\alpha, \beta ; \gamma ; z) \\
2 i a z^{\mu+1 / 2}(1-z)^{\nu} F(\alpha, \beta+1 ; \gamma+1 ; z)
\end{array}\right), \\
z=-e^{-2 i \varphi}, \mu=\frac{c-i b}{2 \omega}, \nu=\frac{i}{\omega} \sqrt{a^{2}+b^{2}}, \\
\alpha=\frac{1}{2}+\frac{c}{\omega}+\nu, \beta=\nu-\frac{i b}{\omega}, \gamma=\frac{1}{2}+2 \mu .
\end{array}
$$

8. $F_{1}=a / \sinh \varphi, F_{3}=b \tanh \varphi+c \operatorname{coth} \varphi$ :

$$
\begin{array}{r}
V=\left(\begin{array}{r}
-a z^{\mu}(1-z)^{\nu} F(\alpha, \beta ; \gamma ; z) \\
(-2 i \omega \mu a+c) z^{\mu}(1-z)^{\nu+1 / 2} F(\alpha, \beta+1 ; \gamma ; z)
\end{array}\right), \\
z=\tanh ^{2} \varphi, \mu=\frac{i}{2 \omega} \sqrt{a^{2}+c^{2}}, \nu=\frac{i(b+c)}{2 \omega}, \\
\alpha=\frac{1}{2}+\frac{i b}{\omega}+\beta, \beta=\mu+\frac{i c}{2 \omega}, \gamma=2 \mu+1 .
\end{array}
$$


9. $F_{1}=a / \cosh \varphi, F_{3}=b \tanh \varphi+c \operatorname{coth} \varphi:$

$$
\begin{array}{r}
V=\left(\begin{array}{l}
(2 c+i \omega) z^{\mu}(1-z)^{\nu} F(\alpha, \beta ; \gamma ; z) \\
a z^{\mu+1 / 2}(1-z)^{\nu+1 / 2} F(\alpha+1, \beta+1 ; \gamma+1 ; z)
\end{array}\right), \\
z=\tanh ^{2} \varphi, \mu=-\frac{i c}{2 \omega}, \nu=\frac{i(b+c)}{2 \omega}, \lambda=\frac{1}{2 \omega} \sqrt{a^{2}-b^{2}}, \\
\alpha=\frac{i b}{2 \omega}+\lambda, \beta=\frac{i b}{2 \omega}-\lambda, \gamma=\frac{1}{2}-\frac{i c}{\omega} .
\end{array}
$$

10. $F_{1}=a / \sinh 2 \varphi, F_{3}=(b \cosh 2 \varphi+c) / \sinh 2 \varphi:$

$$
\begin{aligned}
& V=\left(\begin{array}{l}
-a z^{\mu}(1-z)^{\nu} F(\alpha, \beta ; \gamma ; z) \\
(-4 i \omega \mu+b+c) z^{\mu}(1-z)^{\nu+1} F(\alpha+1, \beta+1 ; \gamma ; z)
\end{array}\right) \\
& z=\tanh ^{2} \varphi, \mu=\frac{i}{4 \omega} \sqrt{a^{2}+(b+c)^{2}}, \lambda=\frac{i}{4 \omega} \sqrt{a^{2}+(b-c)^{2}} \\
& \nu=\frac{i b}{2 \omega}, \alpha=\mu+\nu+\lambda, \beta=\mu+\nu-\lambda, \gamma=1+2 \mu
\end{aligned}
$$

11. $F_{1}=a / \cosh \varphi, F_{3}=(b \sinh \varphi+c) / \cosh \varphi:$

$$
\begin{gathered}
V=\left(\begin{array}{l}
a z^{\mu}(1-z)^{\nu} F(\alpha, \beta ; \gamma ; z) \\
(2 \omega \mu-c+i b) z^{\mu}(1-z)^{\nu+1} F(\alpha+1, \beta+1 ; \gamma ; z)
\end{array}\right), \\
z=\left(\frac{e^{\varphi}+i}{e^{\varphi}-i}\right)^{2}, \mu=\frac{1}{2 \omega} \sqrt{a^{2}+(c-i b)^{2}}, \alpha=\mu+\nu+\lambda, \\
\lambda=\frac{1}{2 \omega} \sqrt{a^{2}+(c+i b)^{2}}, \nu=\frac{i b}{\omega}, \beta=\mu+\nu-\lambda, \gamma=1+2 \mu .
\end{gathered}
$$

12. $F_{1}=a \tanh \varphi, F_{3}=b \tanh \varphi+c \operatorname{coth} \varphi:$

$$
\begin{array}{r}
V=\left(\begin{array}{c}
2(c+i \omega) z^{\mu}(1-z)^{\nu} F(\alpha, \beta ; \gamma ; z) \\
a z^{\mu+1}(1-z)^{\nu} F(\alpha+1, \beta+1 ; \gamma+2 ; z)
\end{array}\right), \\
z=\tanh ^{2} \varphi, \mu=-\frac{i c}{2 \omega}, \nu=\frac{i}{2 \omega} \sqrt{a^{2}+(b+c)^{2}}, \\
\lambda=\frac{i}{2 \omega} \sqrt{a^{2}+b^{2}}, \alpha=\mu+\nu+\lambda, \beta=\mu+\nu-\lambda, \gamma=2 \mu .
\end{array}
$$


13. $F_{1}=a \operatorname{coth} \varphi, F_{3}=b \tanh \varphi+c \operatorname{coth} \varphi$ :

$$
\begin{array}{r}
V=\left(\begin{array}{l}
-a z^{\mu}(1-z)^{\nu} F(\alpha+1, \beta ; \gamma ; z) \\
(2 \omega \mu+c) z^{\mu}(1-z)^{\nu} F(\alpha, \beta+1 ; \gamma ; z)
\end{array}\right), \\
z=\tanh ^{2} \varphi, \mu=\frac{i}{2 \omega} \sqrt{a^{2}+c^{2}}, \nu=\frac{i}{2 \omega} \sqrt{a^{2}+(b+c)^{2}}, \\
\alpha=\mu+\nu+\frac{i b}{2 \omega}, \beta=\mu+\nu-\frac{i b}{2 \omega}, \gamma=1+2 \mu .
\end{array}
$$

14. $F_{1}=a / \cosh \varphi, F_{3}=b \tanh \varphi+c$ :

$$
\begin{array}{r}
V=\left(\begin{array}{l}
(2 b+2 c-i \omega) z^{\mu}(1-z)^{\nu} F(\alpha, \beta ; \gamma ; z) \\
2 a z^{\mu+1 / 2}(1-z)^{\nu+1 / 2} F(\alpha+1, \beta+1, \gamma+1 ; z)
\end{array}\right), \\
z=\frac{1}{2}(1-\tanh \varphi), \alpha=\mu+\nu+\lambda, \beta=\mu+\nu-\lambda, \\
\mu=\frac{i(b+c)}{2 \omega}, \nu=\frac{i(b-c)}{2 \omega}, \gamma=1 / 2+2 \mu, \lambda=\frac{1}{\omega} \sqrt{a^{2}-b^{2}} .
\end{array}
$$

15. $F_{1}=a / \sinh \varphi, F_{3}=b \operatorname{coth} \varphi+c:$

$$
\begin{array}{r}
V=\left(\begin{array}{l}
-a z^{\mu}(1-z)^{\nu} F(\alpha, \beta ; \gamma ; z) \\
(-i \omega \mu+b) z^{\mu}(1-z)^{\nu+1 / 2} F(\alpha, \beta+1 ; \gamma ; z)
\end{array}\right), \\
z=1-e^{-2 \varphi}, \mu=\frac{i}{\omega} \sqrt{a^{2}+b^{2}}, \nu=\frac{i(b+c)}{2 \omega}, \\
\alpha=\frac{1}{2}+\mu+\frac{i c}{\omega}, \beta=\mu+\frac{i b}{\omega}, \gamma=1+2 \mu .
\end{array}
$$

16. $F_{1}=a, F_{3}=b t+c$ :

$$
\begin{gathered}
V=\left(\begin{array}{l}
2 \sqrt{b} D_{\mu}(z) \\
(1+i) a D_{\mu-1}(z)
\end{array}\right), \\
z=\frac{1+i}{\sqrt{b}}(b t+c), \mu=-\frac{i a^{2}}{2 b} .
\end{gathered}
$$


17. $F_{1}=a, F_{3}=b / t+c:$

$$
\begin{array}{r}
V=\left(\begin{array}{l}
(1-2 i b) t^{\gamma} e^{-z / 2} \Phi(\alpha, 2 \gamma ; z) \\
-i a t^{\gamma+1} e^{-z / 2} \Phi(\alpha+1,2 \gamma+2, z)
\end{array}\right), \\
z=2 i t \sqrt{a^{2}+c^{2}}, \gamma=-i b, \alpha=\gamma\left(1-\frac{c}{\sqrt{a^{2}+c^{2}}}\right) .
\end{array}
$$

18. $F_{1}=a, F_{3}=b / t+c t$ :

$$
\begin{array}{r}
V=\left(\begin{array}{c}
(2 b+i) t^{\gamma-1 / 2} e^{-z / 2} \Phi(\alpha, \gamma ; z) \\
a t^{\gamma+1 / 2} e^{-z / 2} \Phi(\alpha+1, \gamma+1 ; z)
\end{array}\right), \\
z=i c t^{2}, \alpha=\frac{i a^{2}}{4 c}, \gamma=\frac{1}{2}-i b .
\end{array}
$$

19. $F_{1}=a, F_{3}=(b \cos 2 \varphi+c) / \sin 2 \varphi$ :

$$
\begin{gathered}
V=\left(\begin{array}{c}
(b+c+i \omega) z^{\mu}(1-z)^{\nu} F(\alpha, \beta ; \gamma ; z) \\
a z^{\mu+1 / 2}(1-z)^{\nu+1 / 2} F(\alpha+1, \beta+1 ; \gamma+1 ; z)
\end{array}\right), \\
z=\sin ^{2} \varphi, \mu=-\frac{i}{4 \omega}(b+c), \nu=\frac{i}{4 \omega}(c-b), \gamma=\frac{1}{2}+2 \mu, \\
\alpha=\frac{1}{2 \omega}\left(\sqrt{a^{2}-b^{2}}-i b\right), \beta=-\frac{1}{2 \omega}\left(\sqrt{a^{2}-b^{2}}+i b\right) .
\end{gathered}
$$

20. $F_{1}=a, F_{3}=b \tan \varphi+c \cot \varphi:$

$$
\begin{array}{r}
V=\left(\begin{array}{l}
(2 c+i \omega) z^{\mu}(1-z)^{\nu} F(\alpha, \beta ; \gamma ; z) \\
a z^{\mu+1 / 2}(1-z)^{\nu+1 / 2} F(\alpha+1, \beta+1 ; \gamma+1 ; z)
\end{array}\right), \\
z=\sin ^{2} \varphi, \mu=-\frac{i c}{2 \omega}, \nu=\frac{i b}{2 \omega}, \lambda=\frac{1}{2 \omega} \sqrt{a^{2}-(b-c)^{2}}, \\
\alpha=\mu+\nu+\lambda, \beta=\mu+\nu-\lambda, \gamma=\frac{1}{2}+2 \mu .
\end{array}
$$

21. $F_{1}=a, F_{3}=b \tan \varphi+c$ :

$$
\begin{array}{r}
V=\left(\begin{array}{l}
a z^{\mu}(1-z)^{\nu} F(\alpha, \beta ; \gamma ; z) \\
(2 \omega \mu-c+i b) z^{\mu}(1-z)^{\nu+1} F(\alpha+1, \beta+1 ; \gamma ; z)
\end{array}\right), \\
z=-e^{-2 i \varphi}, \mu=\frac{1}{2 \omega} \sqrt{a^{2}+(c-i b)^{2}}, \alpha=\mu+\nu+\lambda, \\
\nu=\frac{i b}{\omega}, \beta=\mu+\nu-\lambda, \gamma=1+2 \mu, \lambda=\frac{1}{2 \omega} \sqrt{a^{2}+(c+i b)^{2}} .
\end{array}
$$


22. $F_{1}=a, F_{3}=b \tanh \varphi+c \operatorname{coth} \varphi:$

$$
\begin{array}{r}
V=\left(\begin{array}{c}
(2 c+i \omega) z^{\mu}(1-z)^{\nu} F(\alpha, \beta ; \gamma ; z) \\
a z^{\mu+1 / 2}(1-z)^{\nu} F(\alpha, \beta+1 ; \gamma+1 ; z)
\end{array}\right), \\
z=\tanh ^{2} \varphi, \mu=-\frac{i c}{2 \omega}, \nu=\frac{i}{2 \omega} \sqrt{a^{2}+(b+c)^{2}}, \\
\gamma=\frac{1}{2}+2 \mu, \alpha=\gamma+\nu+\frac{i}{2 \omega}(b+c), \beta=\nu-\frac{i}{2 \omega}(b+c) .
\end{array}
$$

23. $F_{1}=a, F_{3}=(b \cosh 2 \varphi+c) / \sinh 2 \varphi:$

$$
\begin{array}{r}
V=\left(\begin{array}{c}
(b+c+i \omega) z^{\mu}(1-z)^{\nu} F(\alpha, \beta ; \gamma ; z) \\
a z^{\mu+1 / 2}(1-z)^{\nu} F(\alpha, \beta+1 ; \gamma+1 ; z)
\end{array}\right), \\
z=\tanh ^{2} \varphi, \mu=-\frac{i(b+c)}{4 \omega}, \nu=\frac{i}{2 \omega} \sqrt{a^{2}+b^{2}}, \\
\alpha=\frac{1}{2}+\nu-\frac{i c}{2 \omega}, \beta=\nu-\frac{i b}{2 \omega}, \gamma=\frac{1}{2}+2 \mu .
\end{array}
$$

24. $F_{1}=a, F_{3}=(b \sinh \varphi+c) / \cosh \varphi:$

$$
\begin{array}{r}
V=\left(\begin{array}{c}
(2 b+2 i c+i \omega) z^{\mu}(1-z)^{\nu} F(\alpha, \beta ; \gamma ; z) \\
2 a z^{\mu+1 / 2}(1-z)^{\nu} F(\alpha, \beta+1 ; \gamma+1 ; z)
\end{array}\right), \\
z=\left(\frac{e^{\varphi}+i}{e^{\varphi}-i}\right)^{2}, \mu=\frac{c-i b}{2 \omega}, \nu=\frac{i}{\omega} \sqrt{a^{2}+b^{2}}, \\
\alpha=\frac{1}{2}+\nu+\frac{c}{\omega}, \beta=\nu-\frac{i b}{\omega}, \gamma=\frac{1}{2}+2 \mu .
\end{array}
$$

25. $F_{1}=a, F_{3}=b \tanh \varphi+c:$

$$
\begin{gathered}
V=\left(\begin{array}{c}
a z^{\mu}(1-z)^{\nu} F(\alpha+1, \beta ; \gamma ; z) \\
-(i 2 \omega \mu+b+c) z^{\mu}(1-z)^{\nu} F(\alpha, \beta+1 ; \gamma ; z)
\end{array}\right), \\
z=\frac{1}{2}(1-\tanh \varphi), \alpha=\mu+\nu+\frac{i b}{\omega}, \nu=\frac{i}{2 \omega} \sqrt{a^{2}+(b-c)^{2}}, \\
\beta=\mu+\nu-\frac{i b}{\omega}, \gamma=1+2 \mu, \mu=\frac{i}{2 \omega} \sqrt{a^{2}+(b+c)^{2}} .
\end{gathered}
$$


26. $F_{1}=a, F_{3}=b \operatorname{coth} \varphi+c$ :

$$
\begin{array}{r}
V=\left(\begin{array}{c}
2(2 b+i \omega) z^{\mu}(1-z)^{\nu} F(\alpha, \beta ; \gamma ; z) \\
a z^{\mu+1}(1-z)^{\nu} F(\alpha+1, \beta+1 ; \gamma+2 ; z)
\end{array}\right), \\
z=1-e^{-2 \varphi}, \mu=-\frac{i b}{\omega}, \nu=\frac{i}{2 \omega} \sqrt{a^{2}+(b+c)^{2}}, \alpha=\nu-\frac{i b}{\omega}+\lambda \\
\beta=\nu-\frac{i b}{\omega}-\lambda, \gamma=-\frac{2 i b}{\omega}, \lambda=\frac{i}{2 \omega} \sqrt{a^{2}+(b-c)^{2}} .
\end{array}
$$




\section{Capítulo 8}

\section{Transformações de Darboux}

Considere dois operadores lineares $\hat{h}_{0}$ e $\hat{h}_{1}$, e vamos supor que exista um operador $\hat{L}$ tal que

$$
\hat{L} \hat{h}_{0}=\hat{h}_{1} \hat{L} .
$$

Este operador $\hat{L}$ é chamado operador de entrelaçamento (intertwining operator) dos operadores $\hat{h}_{0}$ e $\hat{h}_{1}$. O conhecimento de $\hat{L}$ permite, a partir de uma solução $V$ do problema de autovalores de $\hat{h}_{0}$,

$$
\hat{h}_{0} V=\varepsilon V,
$$

construir o vetor

$$
V^{\prime}=\hat{L} V
$$

que representa uma solução do problema de autovetores de $\hat{h}_{1}$ com o mesmo autovalor $\varepsilon$,

$$
\hat{h}_{1} V^{\prime}=\varepsilon V^{\prime} .
$$

Quando os operadores $\hat{h}_{0}$ e $\hat{h}_{1}$ são operadores diferenciais e $\hat{L}$ é um operador diferencial de primeira ordem, a relação (8.3) recebe o nome de transformação de Darboux. Este procedimento, que hoje possui inúmeras aplicações, foi utilizado há mais de um século por Darboux no tratamento do problema de Sturm-Liouville [24]. Anos mais tarde, este método atraiu enorme atenção graças a sua aplicação no tratamento de equações diferenciais não lineares [27] e no problema inverso do espalhamento [8]. 


\subsection{Construção do operador de entrelaçamento}

Vamos supor que os vetores $V$ e $V^{\prime}$ representam matrizes coluna com $n$ componentes dependentes do tempo, e $\hat{h}_{0}$ e $\hat{h}_{1}$ são matrizes $n \times n$ cujos elementos contém apenas operadores de derivação de primeira ordem. Neste caso, temos dois sistemas lineares de equações diferenciais de primeira ordem. Seguindo [25], vamos considerar $\hat{h}_{0}$ e $\hat{h}_{1}$ na forma

$$
\hat{h}_{0}=\gamma \frac{d}{d t}+\Lambda_{0}(t), \hat{h}_{1}=\gamma \frac{d}{d t}+\Lambda_{1}(t)
$$

onde $\Lambda_{0}(t)$ e $\Lambda_{1}(t)$ são campos externos matriciais e $\gamma$ uma matriz constante, todas $n \times n$. Neste caso, podemos supor a seguinte forma para o operador de entrelaçamento:

$$
\hat{L}=A(t) \frac{d}{d t}+B(t)
$$

onde $A(t)$ e $B(t)$ são matrizes $n \times n$ dependentes do tempo. A propriedade de entrelaçamento (8.1) do operador $\hat{L}$ resulta no seguinte conjunto de equações para $A(t)$ e $B(t)$,

$$
\begin{aligned}
& A \gamma-\gamma A=0 \\
& \gamma \dot{A}+\gamma B-B \gamma+\Lambda_{1} A-A \Lambda_{0}=0, \\
& \gamma \dot{B}+\Lambda_{1} B-A \dot{\Lambda}_{0}-B \Lambda_{0}=0
\end{aligned}
$$

onde o ponto indica derivação com relação a $t$ e a derivada de uma matriz é a derivada de cada um de seus elementos. Selecionando $A$ como uma matriz não singular, podemos usar (8.8) para definir $\Lambda_{1}$,

$$
\Lambda_{1}=\left(A \Lambda_{0}+B \gamma-\gamma B-\gamma \dot{A}\right) A^{-1}
$$

Substituindo a expressão acima em (8.9) obtemos

$$
\gamma \dot{B}+\left(A \Lambda_{0}+B \gamma-\gamma B-\gamma \dot{A}\right) A^{-1} B-A \dot{\Lambda}_{0}-B \Lambda_{0}=0
$$

onde $A$ permanece uma matriz arbitrária não singular, que obedece à relação (8.7). Podemos eliminar $A$ da expressão acima fazendo

$$
B=A D
$$


para uma certa matriz $D$, com o que (8.11) torna-se

$$
\gamma \dot{D}+\Lambda_{0} D+D \gamma D-\gamma D^{2}-\dot{\Lambda}_{0}-D \Lambda_{0}=0
$$

Em seguida, podemos eliminar o termo em $D^{2}$ fazendo

$$
D=-\dot{\Gamma} \Gamma^{-1} \Longrightarrow B=-A \dot{\Gamma} \Gamma^{-1}
$$

com $\Gamma(t)$ uma matriz arbitrária não singular. Como resultado, obtemos a expressão

$$
A \Gamma \dot{M} \Gamma^{-1}=0,
$$

onde a matriz $M(t)$ obedece à relação

$$
\gamma \dot{\Gamma}+\Lambda_{0} \Gamma=\Gamma M \Longleftrightarrow \hat{h}_{0} \Gamma=\Gamma M .
$$

Podemos satisfazer a equação (8.14), e conseqüentemente (8.9), fazendo $M(t)$ igual a uma matriz constante $(\dot{M}=0)$. Com isto, podemos usar (8.15) como a definição da matriz $\Gamma$. Finalmente, podemos expressar a matriz $B$ e o campo externo transformado $\Lambda_{1} \mathrm{em}$ termos das matrizes $\Gamma, M$ e $A$,

$$
\begin{aligned}
& B=A \gamma^{-1}\left(\Lambda_{0}-\Gamma M \Gamma^{-1}\right), \\
& \Lambda_{1}=A\left(\gamma^{-1} \Lambda_{0} \gamma+\Gamma M \Gamma^{-1}-\gamma^{-1} \Gamma M \Gamma^{-1} \gamma\right) A^{-1}-\gamma \dot{A} A^{-1} .
\end{aligned}
$$

Escolhendo $M$ como uma matriz diagonal,

$$
M_{r s}=\delta_{r s} \lambda_{(s)},
$$

(sem somatória em $s$ ) cada coluna da expressão (8.15) fornecerá uma equação com a mesma forma de (8.2), o que resultará em $n$ equações, para cada um dos $n$ vetores coluna $V_{(1)}, V_{(2)}, \ldots, V_{(n)}$,

$$
\hat{h}_{0} V_{(s)}=\lambda_{(s)} V_{(s)}, V_{(s)}=\left(\begin{array}{c}
\Gamma_{1 s} \\
\Gamma_{2 s} \\
\vdots \\
\Gamma_{n s}
\end{array}\right), s=1,2, \ldots, n
$$


Como resultado, conhecendo-se $n$ soluções diferentes $\left(\lambda_{(s)}, V_{(s)}\right)$ do problema de autovalores de $\hat{h}_{0}(8.2)$, podemos construir a matriz $\Gamma$ fazendo

$$
\Gamma=\left(\begin{array}{llll}
V_{(1)} & V_{(2)} & \cdots & V_{(n)}
\end{array}\right) .
$$

Uma vez construída a matriz $\Gamma$, e fixada a matriz $A$, podemos obter a matriz $B$ e o campo externo $\Lambda_{1}$ de (8.16) e (8.17) e, conseqüentemente, podemos construir o operador de entrelaçamento $\hat{L}$ como

$$
\hat{L}=A \gamma^{-1}\left(\hat{h}_{0}-\Gamma M \Gamma^{-1}\right) .
$$

Assim, dado um autovetor $V$ do problema (8.2), com autovalor $\varepsilon \neq \lambda_{(s)}$, podemos construir um autovetor $V^{\prime}$ do problema (8.4), com o mesmo autovalor $\varepsilon$, fazendo

$$
V^{\prime}=\hat{L} V=A \gamma^{-1}\left(\varepsilon-\Gamma M \Gamma^{-1}\right) V .
$$

Para o caso $\lambda_{(s)}=\varepsilon$ o operador de entrelaçamento aniquila o autovetor correspondente $\hat{L} V^{(\varepsilon)}=0$.

Por substituição direta pode-se verificar que se $V^{\prime}$ é uma solução de (8.4), então $A V^{\prime}$ também o será, de sorte que podemos, sem perda de generalidade, fazer $A=I$, onde $I$ é a matriz identidade $n \times n$.

O método descrito acima pode ser usado iterativamente, de forma que soluções para um novo problema, com um novo campo externo $\Lambda_{2}$, podem ser construídas aplicando-se o mesmo procedimento nos autovetores obtidos de $\hat{h}_{1}$.

\subsection{Transformações de Darboux para a equação de spin}

Nosso objetivo é utilizar o método de Darboux para obter novas soluções da equação de spin com um campo externo na forma

$$
\mathbf{F}_{\varepsilon}=\left(F_{1}, 0, F_{3}\right), F_{1}=\varepsilon=\mathrm{const}, F_{3}=F_{3}(t)=F_{3}^{*} .
$$


Soluções exatas para campos deste tipo podem ser encontradas nos itens 16 - 26 do capítulo anterior. Equações de spin com campos desse tipo aparecem em vários problemas em física $[17,14,26]$. Neste caso, escrevendo a equação de spin na forma (8.2) o operador $\hat{h}_{0}(8.5)$ torna-se

$$
\hat{h}_{0}=i \sigma_{1} \frac{d}{d t}+\Lambda_{0}, \Lambda_{0}=i \sigma_{2} F_{3} .
$$

Desejamos também que, após a aplicação da transformação de Darboux, o novo campo externo obtido possua a mesma forma de (8.23) com uma nova função real $F_{3}^{\prime}$, ou seja, procuramos por operadores de entrelaçamento $\hat{L}$ tais que

$$
\hat{L} \hat{h}_{0}=\hat{h}_{1} \hat{L}, \hat{h}_{1}=i \sigma_{1} \frac{d}{d t}+\Lambda_{1}, \Lambda_{1}=i \sigma_{2} F_{3}^{\prime} .
$$

Existe um método geral para a construção do operador de entrelaçamento para um dado problema de autovalores [27]. Entretanto, para nossos propósitos, a aplicação direta deste método geral não pode ser utilizada. A aplicação deste método destruiria a estrutura inicial da matriz $\Lambda_{0}$ em (8.24), de forma que o novo campo externo obtido $\Lambda_{1}$, em geral, não possuiria a estrutura especificada em $(8.25)$, com uma nova função real $F_{3}^{\prime}$. Então, a peculiaridade de nosso problema é que os campos externos $\Lambda_{0}$ e $\Lambda_{1}$ devem obedecer algumas restrições algébricas e a transformação de Darboux precisa respeitar estas restrições.

A relação de entrelaçamento (8.1), com o operador $\hat{L}$ na forma $(8.6)(\operatorname{com} A=I)$ e o campo $\Lambda_{1}$ na forma (8.25) fornece as seguintes relações algébricas

$$
\begin{aligned}
& \sigma_{1} B-B \sigma_{1}+\sigma_{2}\left(F_{3}^{\prime}-F_{3}\right)=0, \\
& \sigma_{1} \dot{B}+\sigma_{2} B F_{3}^{\prime}-\sigma_{2} \dot{F}_{3}-B \sigma_{2} F_{3}=0 .
\end{aligned}
$$

Vamos escolher

$$
B=\alpha+i\left(F_{3}-\beta\right) \sigma_{3}
$$

para certas funções do tempo $\alpha(t)$ e $\beta(t)$. Então, obteremos para a função $F_{3}^{\prime}$ :

$$
F_{3}^{\prime}=2 \beta-F_{3}
$$

Substituindo (8.29) e (8.28) em (8.27) temos

$$
\dot{\alpha}-2 \beta\left(F_{3}-\beta\right)=0, \dot{\beta}+2 \alpha\left(F_{3}-\beta\right)=0,
$$


de onde segue

$$
\alpha^{2}+\beta^{2}=R^{2}, R=\text { const }
$$

com $R$ uma constante, no caso geral, complexa. Podemos satisfazer a expressão acima fazendo

$$
\alpha=R \cos \mu, \beta=R \sin \mu,
$$

onde $\mu(t)$ é uma função real do tempo. Substituindo (8.32) em (8.30), obtemos para a função $\mu$ uma equação diferencial transcendental

$$
\dot{\mu}=2\left(R \sin \mu-F_{3}\right) .
$$

A seguir, desenvolveremos um método para encontrar as funções $\alpha(t)$ e $\beta(t)$ sem a necessidade de resolver a equação (8.33), conseqüentemente, chegaremos a uma forma indireta de obter soluções para esta equação.

Se $V_{\varepsilon}$ é uma autofunção de $\hat{h}_{0}$, com autovalor $\varepsilon$, a derivada temporal contida no operador $\hat{L}$ (8.6), quando aplicado a $V_{\varepsilon}$, pode ser calculada usando (8.24)

$$
V_{\varepsilon}^{\prime}=\left[\alpha-i\left(\varepsilon \sigma_{1}+\beta \sigma_{3}\right)\right] V_{\varepsilon}
$$

Então, a transformação de Darboux procurada tem a forma (8.34) e pode ser determinada resolvendo-se a equação (8.30) ou (8.33). Entretanto, sabemos da seção anterior que o operador de entrelaçamento pode ser construído a partir do conhecimento de dois autovetores de $\hat{h}_{0}$. Na verdade, é necessário conhecer apenas um autovetor $V_{\varepsilon}$, uma vez que um segundo autovetor $V_{-\varepsilon}$, ortogonal ao primeiro, pode ser construído fazendo $V_{-\varepsilon}=\sigma_{3} V_{\varepsilon}$. Assim, dado um autovetor $V_{\varepsilon_{0}}$ de $\hat{h}_{0}$ com autovalor $\varepsilon_{0}$, podemos construir as matrizes $\Gamma$ (8.20) e $M(8.18)$ como

$$
\Gamma=\left(\begin{array}{cc}
v_{1}^{\varepsilon_{0}} & v_{1}^{\varepsilon_{0}} \\
v_{2}^{\varepsilon_{0}} & -v_{2}^{\varepsilon_{0}}
\end{array}\right), M=\left(\begin{array}{cc}
\varepsilon_{0} & 0 \\
0 & -\varepsilon_{0}
\end{array}\right), V_{\varepsilon_{0}}=\left(\begin{array}{c}
v_{1}^{\varepsilon_{0}} \\
v_{2}^{\varepsilon_{0}}
\end{array}\right) .
$$

Isto permite construir $B$ a partir de (8.16)

$$
B=\gamma^{-1}\left(\Lambda_{0}-\Gamma M \Gamma^{-1}\right)=-\varepsilon_{0} \frac{L_{2}}{L_{3}}+i\left(F_{3}+\varepsilon_{0} \frac{L_{1}}{L_{3}}\right) \sigma_{3}
$$


onde $L_{i} i=1,2,3$ são as componentes do vetor $\mathbf{L}$,

$$
\mathbf{L}=\left(\bar{V}_{\varepsilon_{0}}, \tilde{\sigma} V_{\varepsilon_{0}}\right)
$$

veja (2.24). De acordo com a equação (3.5), este vetor obedece à relação

$$
\dot{\mathbf{L}}=2\left[\mathbf{F}_{\varepsilon_{0}} \times \mathbf{L}\right], \mathbf{F}_{\varepsilon_{0}}=\left(\varepsilon_{0}, 0, F_{3}\right)
$$

Além disto, as equações (2.25) e (2.7) do apêndice implicam em

$$
\mathbf{L}^{2}=0
$$

Comparando (8.36) com (8.28) temos

$$
\alpha=-\varepsilon_{0} \frac{L_{2}}{L_{3}}, \beta=-\varepsilon_{0} \frac{L_{1}}{L_{3}} .
$$

Usando (8.39) e (8.40) temos

$$
\alpha^{2}+\beta^{2}=-\varepsilon_{0}^{2}
$$

além disto, com a ajuda de (8.38), podemos verificar facilmente que (8.40) é uma solução de (8.30) com $\varepsilon_{0}=i R$. Substituindo (8.40) em (8.34), obtemos a forma final da transformação de Darboux procurada,

$$
V_{\varepsilon}^{\prime}=\sigma_{2}\left[(\tilde{\sigma} \mathbf{L}) L_{3}^{-1}+\frac{\varepsilon}{\varepsilon_{0}} \sigma_{3}\right] V_{\varepsilon} .
$$

Usando as equações (2.36) e (B.3) do apêndice, a equação (8.42) pode ser escrita como

$$
V_{\varepsilon}^{\prime}=\left[2\left(\bar{V}_{\varepsilon_{0}}, V_{\varepsilon}\right) L_{3}^{-1} \sigma_{2} V_{\varepsilon_{0}}+i \frac{\varepsilon}{\varepsilon_{0}} \sigma_{1} V_{\varepsilon}\right] .
$$

\subsection{Exemplos do método de Darboux}

\subsubsection{Primeiro exemplo}

Como primeiro exemplo, vamos considerar o caso mais simples da transformação de Darboux para um campo inicial constante, ou seja, $F_{3}=c=$ const. Neste caso, a solução 
geral da equação de spin pode ser obtida de (7.5) e (7.7),

$$
V_{\varepsilon}(t)=\left(\begin{array}{c}
i(c-\omega) p \exp (i \omega t)-\varepsilon q \exp (-i \omega t) \\
i \varepsilon p \exp (i \omega t)+(c-\omega) q \exp (-i \omega t)
\end{array}\right), \omega^{2}=c^{2}+\varepsilon^{2},
$$

onde $p$ e $q$ são constantes complexas arbitrárias. Fazendo $\varepsilon=i R_{0}$ nas soluções acima, as funções $\alpha$ e $\beta$, as quais chamaremos $\alpha_{0}$ e $\beta_{0}$, podem ser facilmente encontradas, de (8.40) temos

$$
\begin{aligned}
& \alpha_{0}(t)=-\frac{\dot{Q}_{0}}{2\left(Q_{0}-c\right)}, \beta_{0}(t)=c+\frac{c^{2}-R_{0}^{2}}{Q_{0}-c}, \\
& Q_{0}(t)=R_{0} \cosh \varphi, \varphi=2\left(\omega_{0} t+\varphi_{0}\right), \omega_{0}^{2}=R_{0}^{2}-c^{2},
\end{aligned}
$$

onde $R_{0}$ e $\varphi_{0}$ são constantes complexas arbitrárias. Com isto, o novo campo externo $F_{3}^{\prime}$ pode ser encontrado de (8.29),

$$
F_{3}^{\prime}(t)=c+2 \frac{c^{2}-R_{0}^{2}}{Q_{0}-c} .
$$

Se $c$ é real, então $F_{3}^{\prime}$ também o será, para uma escolha de $R_{0}$ e $\varphi_{0}$ reais e $R_{0}^{2}>c^{2}$. Substituindo $\varphi_{0}$ por $i \varphi_{0}$, também obteremos $F_{3}^{\prime}$ real, pois, com isto $Q_{0}=R_{0} \cos \varphi$ e $\omega_{0}=$ $\sqrt{\left|R_{0}^{2}-c^{2}\right|}$. Para $c$ imaginário, obteremos um $F_{3}^{\prime}$ imaginário quando $R_{0}$ for imaginário.

As novas soluções $V_{\varepsilon}^{\prime}$ para o campo externo (8.46) podem ser facilmente construídas usando a expressão (8.34),

$$
V_{\varepsilon}^{\prime}=\left(\begin{array}{cc}
\frac{\dot{Q}_{0}}{2\left(Q_{0}-c\right)}-i\left(c+\frac{c^{2}-R_{0}^{2}}{Q_{0}-c}\right) & -i \varepsilon \\
-i \varepsilon & \frac{\dot{Q}_{0}}{2\left(Q_{0}-c\right)}+i\left(c+\frac{c^{2}-R_{0}^{2}}{Q_{0}-c}\right)
\end{array}\right) V_{\varepsilon},
$$

$\operatorname{com} V_{\varepsilon}$ dado por (8.44) e $Q_{0}(t)$ por (8.45).

\subsubsection{Segundo exemplo}

Para ilustrar a iteratividade do método, vamos aplicar novamente a transformação de Darboux no resultado obtido anteriormente. Para isso, fazemos $\varepsilon=i R_{1} \mathrm{em}$ (8.47), com 
$R_{1}$ uma nova constante real, e substituímos o espinor assim obtido em (8.40), o que fornece

$$
\begin{aligned}
\alpha_{1}(t)= & R_{1} S\left[2 \alpha_{0}\left(c_{0} \beta_{0}-R_{1}^{2}\right) Q_{1}+\left(2 \beta_{0}^{2}-R_{0}^{2}-R_{1}^{2}\right) \dot{Q}_{1} / 2+2 R_{1} \alpha_{0}\left(\beta_{0}-c_{0}\right)\right], \\
\beta_{1}(t)= & -R_{1} S\left\{\left[c_{0}\left(2 \beta_{0}^{2}-R_{0}^{2}+R_{1}^{2}\right)-2 \beta_{0} R_{1}^{2}\right] Q_{1}-\alpha_{0} \beta_{0} \dot{Q}_{1}\right. \\
& \left.+R_{1}\left[R_{1}^{2}-R_{0}^{2}+2 \beta_{0}\left(\beta_{0}-c_{0}\right)\right]\right\},
\end{aligned}
$$

onde $\alpha_{0}(t)$ e $\beta_{0}(t)$ são dados por $(8.45)$ e

$$
\begin{aligned}
& S^{-1}=R_{1}\left(R_{0}^{2}+R_{1}^{2}-2 \beta_{0} c_{0}\right) Q_{1}+\alpha_{0} R_{1} \dot{Q}_{1}+\left(R_{0}^{2}+R_{1}^{2}\right) c_{0}-2 \beta_{0} R_{1}^{2}, \\
& Q_{1}=R_{1} \cosh \varphi_{1}, \varphi_{1}=2\left(\omega_{1} t+\gamma_{1}\right), \omega_{1}=\sqrt{\left|R_{1}^{2}-c_{0}^{2}\right|} .
\end{aligned}
$$

com $\gamma_{1}$ uma constante complexa arbitrária. Novamente, as soluções exatas $V_{\varepsilon}^{\prime \prime}$ para o campo externo

$$
F_{3}^{\prime \prime}(t)=2 \beta_{1}(t)+F_{3}^{\prime}(t)=2\left[\beta_{1}(t)+\beta_{0}(t)\right]-c,
$$

com $c$ o campo constante do exemplo anterior, podem ser obtidos substituindo $\alpha_{1}$ e $\beta_{1}$ em (8.34),

$$
V_{\varepsilon}^{\prime \prime}=\left[\alpha_{1}-i\left(\varepsilon \sigma_{1}+\beta_{1} \sigma_{3}\right)\right] V_{\varepsilon}^{\prime},
$$

onde $V_{\varepsilon}^{\prime}$ são os espinores (8.47).

\subsubsection{Terceiro exemplo}

Vamos construir a transformação de Darboux para um campo $F_{3}$ na forma

$$
F_{3}(t)=\frac{r_{0}}{T} \tanh \tau+\frac{r_{1}}{T}, \tau=\frac{t}{T},
$$

onde $r_{0}, r_{1}$ e $T$ são constantes reais. Soluções da equação de spin para o campo externo acima podem ser escritas como (veja [16])

$$
\begin{aligned}
v_{1}= & (1-z)^{\nu} E\left[c_{1} z^{\mu} F(a+1, b ; c ; z)+c_{2} z^{-\mu} F(\bar{a}+1, \bar{b} ; \bar{c} ; z)\right], \\
v_{2}= & (1-z)^{\nu}\left[\left(r_{0}-r_{1}+2 i \mu\right) c_{1} z^{\mu} F(a, b+1 ; c ; z)+\right. \\
& \left.\left(r_{0}-r_{1}-2 i \mu_{0}\right) c_{2} z^{-\mu} F(\bar{a}, \bar{b}+1 ; \bar{c} ; z)\right]
\end{aligned}
$$


onde

$$
\begin{aligned}
& z=\frac{1}{2}(1+\tanh \tau), a=\mu+\nu+i r_{0}, b=\mu+\nu-i r_{0}, \bar{a}=-\mu+\nu+i r_{0}, \\
& \bar{b}=-\mu+\nu-i r_{0}, c=1+2 \mu, \bar{c}=1-2 \mu, E=\varepsilon T,
\end{aligned}
$$

e $c_{1}$ e $c_{2}$ são constantes complexas. Se a seguinte relação for satisfeita

$$
\begin{aligned}
& 4 \mu^{2}+E^{2}+\left(r_{0}-r_{1}\right)^{2}=0, \\
& 4 \nu^{2}+E^{2}+\left(r_{0}+r_{1}\right)^{2}=0,
\end{aligned}
$$

podemos identificar $F(a, b ; c ; z)$ com as funções hipergeométricas.

Construiremos o operador de entrelaçamento $\hat{L}$ apenas para o caso quando $\mu$ e $\nu$ são reais. Fazendo $E=i R$ em $(8.52)$, a condição de realidade será satisfeita para

$$
R^{2}>\max \left(r_{0} \pm r_{1}\right)^{2}
$$

Neste caso, podemos escrever

$$
\mu_{0}=\frac{1}{2} \sqrt{R^{2}-\left(r_{0}-r_{1}\right)^{2}}, \nu_{0}=\frac{1}{2} \sqrt{R^{2}-\left(r_{0}+r_{1}\right)^{2}},
$$

e as expressões (8.51) tornam-se

$$
\begin{aligned}
& v_{1}^{(0)}=-i R(1-z)^{\nu_{0}}\left(c_{1} z^{\mu_{0}} F_{0}+c_{2} z^{-\mu_{0}} F_{1}\right), \\
& v_{2}^{(0)}=(1-z)^{\nu_{0}}\left[\left(r_{0}-r_{1}+2 i \mu_{0}\right) c_{1} z^{\mu_{0}} F_{0}^{*}+\left(r_{0}-r_{1}-2 i \mu_{0}\right) c_{2} z^{-\mu_{0}} F_{1}^{*}\right], \\
& F_{0}=F\left(a_{0}+1, a_{0}^{*} ; 1+2 \mu_{0} ; z\right), \quad F_{1}=F\left(\bar{a}_{0}+1, \bar{a}_{0}^{*} ; 1-2 \mu_{0} ; z\right), \\
& a_{0}=\mu_{0}+\nu_{0}+i r_{0}, \quad \bar{a}=-\mu_{0}+\nu_{0}+i r_{0} .
\end{aligned}
$$

Escolhendo as constantes $c_{1}$ e $c_{2}$ satisfazendo a relação

$$
\frac{c_{1}}{c_{2}}=p^{2 \mu_{0}}\left(r_{0}-r_{1}-2 i \mu_{0}\right) R^{-1}=p^{2 \mu_{0}} e^{-2 i \varphi_{0}}
$$

com $p$ uma nova constante real e $\varphi_{0}$ uma fase constante definida, de acordo com (8.53), pela expressão

$$
\left(r_{0}-r_{1}+2 i \mu_{0}\right) R^{-1}=e^{2 i \varphi_{0}}
$$


Para tal escolha de $c_{1}$ e $c_{2}$ as soluções (8.54) assumem a forma

$$
\begin{aligned}
& v_{1}^{(0)}=-i R(1-z)^{\nu_{0}} \sqrt{c_{1} c_{2}} A, v_{2}^{(0)}=R(1-z)^{\nu_{0}} \sqrt{c_{1} c_{2}} A^{*}, \\
& A=(p z)^{\mu_{0}} e^{-i \varphi_{0}} F_{0}+(p z)^{-\mu_{0}} e^{i \varphi_{0}} F_{1} .
\end{aligned}
$$

Substituindo as soluções acima em (8.40) obtemos as seguintes expressões para $\alpha$ e $\beta$ :

$$
\alpha=\frac{i R\left(A^{* 2}-A^{2}\right)}{2 T A A^{*}}, \beta=F_{3}+\frac{R\left(A^{* 2}+A^{2}\right)}{2 T A A^{*}},
$$

com $F_{3}$ dado por (8.50). Assim, as soluções $V_{\varepsilon}^{\prime}$ exatas para o potencial

$$
F_{3}^{\prime}=\frac{R\left(A^{* 2}+A^{2}\right)}{T|A|^{2}}-\frac{r_{0}}{T} \tanh \tau-\frac{r_{1}}{T},
$$

podem ser obtidas da expressão (8.34) $\operatorname{com} \alpha$ e $\beta$ dados por (8.58). 


\section{Capítulo 9}

\section{Conclusão}

A equação de spin e o seu estudo detalhado são assuntos importantes, uma vez que vários problemas em física podem ser descritos e analisados através desta equação. Além disto, é de grande interesse encontrar soluções exatas desta equação para diferentes campos externos, pois tais soluções permitem modelar o comportamento de sistemas reais, ou seja, implementáveis em laboratório. Em particular, a construção de portas lógicas quânticas, como a porta universal de Hadamard, pode ser realizada fisicamente como um sistema de dois níveis e, conseqüentemente, descrita pela equação de spin.

Na construção de soluções exatas da equação de spin, mostramos como é possível explorar a riqueza de vários outros problemas fisicamente equivalentes e cujas soluções são conhecidas. Outrossim, desenvolvemos o método das transformações de Darboux para sistemas de dois níveis, com o qual podemos utilizar as soluções previamente obtidas com um determinado campo externo, para gerar novas soluções com campos diferentes.

Durante a construção do operador de entrelaçamento, para a aplicação das transformações de Darboux, demonstramos o importante e não trivial fato da existência de uma classe de transformações sob as quais as equações para os sistemas de dois níveis têm sua forma invariante, i.e., a forma matricial do campo externo inicial não se altera após a aplicação desta transformação. Além disto, apresentamos um método direto, e de simples aplicação, para a obtenção explícita de elementos desta classe. Este método pode ser 
diretamente aplicado às soluções 16 a 26 de nossa lista de soluções e permite obter uma infinidade de novos campos e suas soluções exatas. É importante notar que alguns destes campos externos são dados por funções não-periódicas e o tratamento destes campos pelos métodos aproximativos usuais é, em geral, bastante problemático.

Entre os futuros desenvolvimentos deste trabalho, pretendemos criar uma generalização dos resultados aqui presentes para a descrição do sistema de quatro níveis de dois spins interagentes e, com isto, obter a realização de portas lógicas quânticas de dois qubits, essenciais para a implementação das portas lógicas universais. 


\section{Apêndice A}

\section{O problema de autovalores}

Vamos considerar o problema geral de autovalores num espaço de espinores bidimensionais:

$$
A V=\Lambda V
$$

Onde $A$ é uma dada matriz $2 \times 2$ e $\Lambda$ um autovalor. Para soluções não triviais, temos a condição

$$
\operatorname{det}(A-\Lambda I)=0
$$

da qual segue

$$
\operatorname{det}(A-\Lambda I)=\Lambda^{2}-\Lambda \operatorname{tr} A+\operatorname{det} A
$$

Com a expressão acima, vemos que a equação (A.2) implica na possibilidade de dois autovalores não nulos,

$$
\Lambda_{\zeta}=\frac{1}{2}\left[\operatorname{tr} A+\zeta \sqrt{(\operatorname{tr} A)^{2}-4 \operatorname{det} A}\right], \zeta= \pm 1
$$

Por outro lado, toda matriz $2 \times 2 A$ pode ser escrita como

$$
A=a_{0} I+(\tilde{\sigma} \mathbf{a})=\frac{1}{2} \operatorname{tr} A+(\tilde{\sigma} \mathbf{a})
$$

Portanto, o problema de autovalores (A.1) pode ser escrito como

$$
(\tilde{\sigma} \mathbf{a}) V=\lambda V
$$


onde $\lambda=\Lambda-\frac{1}{2} \operatorname{tr} A$.

Para $\mathbf{a}^{2} \neq 0$, existem duas soluções não triviais (uma vez que $\operatorname{det}(\tilde{\sigma} \mathbf{a})=-\mathbf{a}^{2}$ ). Multiplicando (A.6) por $\tilde{\sigma} \mathbf{a}$ pela esquerda, e levando em conta $(\tilde{\sigma} \mathbf{a})(\tilde{\sigma} \mathbf{a})=\mathbf{a}^{2}$, obtemos

$$
\mathbf{a}^{2} V=\lambda^{2} V \Longrightarrow \lambda_{\zeta}=\zeta \sqrt{\mathbf{a}^{2}}, \zeta= \pm 1, \mathbf{a}^{2} \neq 0
$$

O autovetor correspondente $V_{\zeta}$ pode ser escrito como

$$
V_{1}=N_{1}\left(\begin{array}{c}
a_{3}+\sqrt{\mathbf{a}^{2}} \\
a_{1}+i a_{2}
\end{array}\right), V_{-1}=N_{-1}\left(\begin{array}{c}
i a_{2}-a_{1} \\
a_{3}+\sqrt{\mathbf{a}^{2}}
\end{array}\right),
$$

$\operatorname{com} N_{\zeta}$ um fator de normalização.

Para $\mathbf{a}^{2}=0$, a matriz $\tilde{\sigma} \mathbf{a}$ é singular, e existe uma solução não trivial $V_{0}$ da equação (A.6) $\operatorname{para} \lambda=0$,

$$
V_{0}=N\left(\begin{array}{c}
i a_{2}-a_{1} \\
a_{3}
\end{array}\right), \mathbf{a}^{2}=0
$$




\section{Apêndice B}

\section{O problema inverso de autovalores}

Dados dois espinores linearmente independentes $U$ e $V$, o problema inverso de autovalores consiste em determinar a existência de um vetor a tal que o problema de autovalores (A.6) admita soluções $V_{\zeta}, \zeta= \pm 1$ da forma

$$
V_{1}=U, V_{-1}=V .
$$

$\mathrm{O}$ vetor a pode ser determinado a partir dos espinores $U$ e $V$ a menos de uma constante multiplicativa $N$,

$$
\mathbf{a}=N \mathbf{L}^{\bar{U}, V},
$$

com o vetor $\mathbf{L}^{\bar{U}, V}$ definido em (2.24). Para provar esta afirmação, considere a matriz $A^{U, V}$,

$$
\begin{aligned}
& A^{U, V}=\tilde{\sigma} \mathbf{L}^{U, V}=2 V U^{+}-(U, V) I \\
& =\left(\begin{array}{cc}
u_{1}^{*} v_{1}-u_{2}^{*} v_{2} & 2 u_{2}^{*} v_{1} \\
2 u_{1}^{*} v_{2} & u_{2}^{*} v_{2}-u_{1}^{*} v_{1}
\end{array}\right) .
\end{aligned}
$$

Esta matriz possui as seguintes propriedades:

$$
\begin{aligned}
& A^{U, V}=\left(A^{V, U}\right)^{+}, \operatorname{det} A^{U, V}=-(U, V)^{2}, \\
& A^{U, V} V=(U, V) V, A^{U, V} \bar{U}=-(U, V) \bar{U}, \\
& U^{+} A^{U, V}=(U, V) U^{+}, \bar{V}^{+} A^{U, V}=-(U, V) \bar{V}^{+} .
\end{aligned}
$$


Portanto, a prova da afirmação segue de (B.3) e (B.4).

Se tomarmos $U$ e $V$ ortogonais,

$$
(U, V)=0 \Longleftrightarrow U=\alpha \bar{V}, \alpha=\text { const },
$$

então (B.2) se reduz a

$$
\mathbf{a}=N^{\prime} \mathbf{L}^{V, V}, N^{\prime}=-\alpha^{*} N,
$$

veja (2.7). Note que o vetor $\mathbf{L}^{V, V}$ é real.

Em particular, as considerações acima nos permitem concluir que os autovetores $U$ e $V$ da equação (A.6) são ortogonais se, e somente se, a é o produto de um fator complexo e um vetor real. Neste caso, os espinores $V$ e $\bar{V}$ obedecem à relação de completeza (2.9). 


\section{Referências Bibliográficas}

[1] Shirley J.H., Solution of the Schrödinger Equation with a Hamiltonian Periodic in Time, Phys. Rev. 138, B979 (1965)

[2] Feynman R.P., Lectures on Physics, Vol. III, Lecture 9, Addison-Wesley Publ. Co. (1965)

[3] Nussenzveig H.M., Introduction to Quantum Optics, (Gordon and Breach, New York 1973)

[4] Rabi I.I., Ramsey N.F., Schwinger J., Use of Rotating Coordinates in Magnetic Resonance Problems, Rev. Mod. Phys. 26, 167 (1945)

[5] Feynman R.P., Vernon F.L., Hellwarth R.W., Geometrical Representation of the Schrödinger Equation for Solving Maser Problems, J. Applied Physics 28, 49 (1957)

[6] Pauli W., Zur Quantenmechanik des magnetischen Elektrons, Zeit. Phys. 43, 601 (1927)

[7] Grifoni M., Hanggi P., Driven quantum tunneling, Phys. Rep. 304, 229 (1998)

[8] Novikov S. et al, Theory of Solitons, (Consultants Bureau, New York, London 1984)

[9] Rabi I.I., Space Quantization in a Gyrating Magnetic Field, Phys. Rev. 51, 652 (1937)

[10] Dion D.R., Hirschfelder J.O., Adv. Chem. Phys. 35, 265 (1976); Aravind P.K., Hirschfelder J.O., Two-state systems in semiclassical and quantized fields, J. Phys. Chem. 88, 4788 (1984) 
[11] Bloch F., Siegert A., Magnetic Resonance for Nonrotating Fields, Phys. Rev. 57, 522 (1940)

[12] Blümel R., Buchleitner A., Graham R., Sirko L., Smilansky U., Walther H., Dynamical localization in the microwave interaction of Rydberg atoms: The influence of noise, Phys. Rev. A 44, 4521 (1991)

[13] Barata J.C., Wreszinski W.F., Strong-Coupling Theory of Two-Level Atoms in Periodic Fields, Phys. Rev. Lett. 84, 2112 (2000)

[14] Barata J.C., Cortez D.A., Time evolution of two-level systems driven by periodic fields, Phys Lett. A 301, 350 (2002)

[15] Magnus W., Winkler S., Hill's Equation, Dover, New York (1979)

[16] Bagrov V.G., Barata J.C.A., Gitman D.M., Wreszinski W.F., Aspects of two-level systems under external time-dependent fields, J. Phys A34, 10869 (2001)

[17] Bagrov V.G., Baldiotti M.C., Gitman D.M., Shamshutdinova V.V., Darboux transformation for two-level system, Ann. Phys. (Leipzig) 14, No 6, 390 (2005)

[18] Barenco A., Bennett C.H., Cleve R., DiVicenzo D.P., Margolus N., Shor P.W., Sleator T., Smolin J., Weinfurter H., Elementary gates for quantum computation,Phys.Rev. A 523457 (1995)

[19] Angelo R.M., Wreszinski W.F., Quantum NOT Operation and Integrability in TwoLevel Systems, quant-ph/0502102v1 (2005)

[20] Aharonov Y., Anandan J., Phase Change during a Cyclic Quantum Evolution, Phys. Rev. Lett. 58 1593-1596 (1987)

[21] Ekert A., Ericsson M., Hayden P., Inamori H., Jones J.A., Oi D.K.L., Vedral V., Geometric Quantum Computation, J. Mod. Opt. 47, No. 14-15, 2501 (2000) 
[22] Gradshtein I.S., Ryzhik N.M., Tables of Integrals, Sums, Series and Products, (Nauka, Moscow 1971)

[23] Bagrov V.G., Gitman D.M., Exact Solutions of Relativistic Wave Equations, (Kluwer, Dordrecht, Boston, London 1990)

[24] Darboux G., Sur une proposition relative aux équations linéaires, C.R. Acad. Sci. 94, $1456(1882)$

[25] Nieto I.M., Pecheritsin A.A., Samsonov B.F., Intertwining technique for the onedimensional stationary Dirac equation, Ann. Phys. 305, No 2, 151 (2003)

[26] Barata. J.C., On Formal Quasi-Periodic Solutions of the Schrödinger Equation for a Two-Level System with a Hamiltonian Depending Quasi-Periodically in Time, Rev. Math. Phys. 12, 25 (2000)

[27] Matveev V.B., Salle M.A., Darboux transformations and solutions, (Springer-Verlag, Berlin 1991) 